OPEN ACCESS

Edited by:

Caisheng Wu,

Xiamen University, China

Reviewed by:

Xiaoyu Xie,

Xi'an Jiaotong University, China Carlo Gabriele Tocchetti, University of Naples Federico II, Italy

${ }^{*}$ Correspondence:

Tomas Jelinek

tomas.jelinek.md@gmail.com

Specialty section: This article was submitted to

Predictive Toxicology,

a section of the journal

Frontiers in Pharmacology

Received: 30 June 2021

Accepted: 03 August 2021

Published: 13 August 2021

Citation:

Hradska $K$, Hajek $R$ and Jelinek $T$ (2021) Toxicity of Immune-Checkpoint Inhibitors in Hematological Malignancies. Front. Pharmacol. 12:733890.

doi: $10.3389 /$ fphar.2021.733890

\section{Toxicity of Immune-Checkpoint Inhibitors in Hematological Malignancies}

\author{
Katarina Hradska ${ }^{1}$, Roman Hajek ${ }^{1,2}$ and Tomas Jelinek ${ }^{1,2 *}$ \\ ${ }^{1}$ Department of Haematooncology, University Hospital Ostrava, Ostrava, Czechia, ${ }^{2}$ Faculty of Medicine, University of Ostrava, \\ Ostrava, Czechia
}

Immune checkpoint inhibitors (ICls), especially those targeting the programmed-death 1 (PD-1) receptor and its ligands, have become indispensable agents in solid tumor anticancer therapy. Concerning hematological malignancies, only nivolumab and pembrolizumab have been approved for the treatment of relapsed and refractory classical Hodgkin lymphoma and primary mediastinal large B cell lymphoma to date. Nevertheless, clinical research in this field is very active. The mechanism of action of ICls is based on unblocking the hindered immune system to recognize and eliminate cancer cells, but that also has its costs in the form of $\mathrm{ICl}$-specific immune related adverse events (irAEs), which can affect any organ system and can even be lethal. In this article, we have reviewed all prospective blood cancer clinical trials investigating ICls (both monotherapy and combination therapy) with available toxicity data with the purpose of determining the incidence of irAEs in this specific setting and to offer a brief insight into their management, as the use of immune checkpoint blockade is not so frequent in hemato-oncology.

Keywords: hematological malignancies, immune checkpoint inhibitors, toxicity, immune related adverse events, PD-1, PD-L1

\section{INTRODUCTION}

In recent years, immunotherapy has become one of the pillars of cancer treatment. Extensive research in cancer biology has led to a deeper understanding of cancer cell survival mechanisms and the importance of the tumor microenvironment. Not only are we gradually uncovering the essential principles of cancerogenesis and immune escape, but we are also using this knowledge therapeutically. One of the most groundbreaking findings is definitely the discovery of tumor cells' exploitation of immune checkpoints and the introduction of immune checkpoint inhibitors (ICIs).

A normally functioning immune system should be able to distinguish the body's own antigens from foreign or mutated self-proteins and this executive role is borne by $\mathrm{T}$ cells (Buchbinder and Desai, 2016; Jelinek and Hajek, 2016). The vital part of the recognition process is the interaction between a T cell receptor (TCR) and a major histocompatibility complex (MHC) on the surface of the antigen presenting cell (APC), which displays the antigen in question. In addition, there are many other co-stimulatory or co-inhibitory pathways (immune checkpoints) that help to determine the fate of the T lymphocyte. Stimulatory pathways lead to T cells' activation, maturation, expansion and the inhibition of their apoptosis, so they can eliminate defective or autoreactive cells, whereas inhibitory pathways have the opposite effect, causing $\mathrm{T}$ cell anergy and exhaustion and inducing tolerance (Buchbinder and Desai, 2016; Jelinek et al., 2018; Hradska et al., 2020). 
The two most important negative regulators of $\mathrm{T}$ cell immune function are cytotoxic $\mathrm{T}$ lymphocyte-associated antigen 4 (CTLA-4) and programmed death 1 (PD-1) immune checkpoints (Buchbinder and Desai, 2016). CTLA-4 helps to prevent autoreactivity in the early stages of immune response, especially in the lymph nodes, and can be found in the cytoplasm of naïve $\mathrm{T}$ cells and on the surface of regulatory $\mathrm{T}$ cells (Tregs). Upon binding to its cognate ligands B7-1 and B7-2 on the APCs, it leads to T cell anergy (Buchbinder and Desai, 2016; Matsuki and Younes, 2016; Jelinek et al., 2017; Hradska et al., 2020). PD-1 is typical for "exhausted" $\mathrm{T}$ cells that have experienced high levels of stimulation, for example, during chronic infection or cancer, leading to their suboptimal function. However, various immune cells such as B cells and natural killer cells also show PD-1 expression. PD-1 ligands PD-L1 and PD-L2 can be found on various hematopoietic (lymphocytes, APCs) and nonhematopoietic cells (endothelial, parenchymatic cells), but are also induced on many types of tumor cells. PD-1/PD-L1/2 pathway is effective later in the immune response, primarily in peripheral tissues and, under normal circumstances, maintains peripheral tolerance during the course of infection and prevents autoimmunity (Buchbinder and Desai, 2016; Wang et al., 2018).

The rationale behind immune checkpoint blockade is to reinvigorate the immune system, which is inhibited by overexpression of immune checkpoint inhibitor molecules on the surface of cancer cells, to restore $\mathrm{T}$ cell cytotoxicity and to destroy defective cells (Jelinek et al., 2018; Hradska et al., 2020). Immune checkpoint inhibitors, monoclonal antibodies (mAbs) targeting PD-1, PD-L1/2 and CTLA-4, were introduced in 2011 and changed the landscape of especially solid tumor cancer treatment so fundamentally that this breakthrough led to the award of the Nobel Prize in 2018 jointly to James P. Allison and Tasuku Honjo (Smyth and Teng, 2018). In hemato-oncology, only two molecules have been approved by the FDA (US Food and Drug Administration) - nivolumab (anti-PD-1 mAb) for the treatment of relapsed/refractory (RR) classical Hodgkin lymphoma (cHL) and pembrolizumab (anti-PD-1 mAb) also for the treatment of RR $\mathrm{CHL}$ and for RR primary mediastinal large B cell lymphoma (PMBL) as well (Ansell et al., 2015; Armand et al., 2016, 2018a, 2018b; Chen et al., 2017). However, this is just the tip of the iceberg as plenty of ICIs are investigated in many other hematological malignancies such as non-Hodgkin lymphomas (NHLs), multiple myeloma (MM), acute myeloid leukemia (AML), myelodysplastic syndrome (MDS) and chronic lymphocytic leukemia (CLL).

The new anti-cancer strategy came with a new specific type of toxicity which is known as immune related adverse events (irAEs) (Haanen et al., 2017; Fan et al., 2021). The pathophysiological mechanism is not yet fully understood, but irAEs are considered to be $\mathrm{T}$ cell mediated and mark the reinvigorated immune system which may cause organ-specific inflammation, tissue damage and autoimmunity (Chhabra and Kennedy, 2021). Activation of the complement may also play a role in the process (Roth et al., 2021). The most frequently affected tissues are skin, gastrointestinal system, endocrine glands, liver and lungs, but irAEs may involve any organ system. They usually occur within weeks to 3 months after the initiation of ICI therapy and are mild to moderate in severity (Haanen et al., 2017). However, lethal cases are also observed, so it is very important for physicians to recognize potential problems early and to take appropriate measures as soon as possible. IrAEs are classified according to the CTCAE (Common Terminology Criteria for Adverse Events) scoring system.

There are many reviews describing ICIs' immune related toxicity in solid tumors or in cancer treatment in general. Nevertheless, ICIs are gradually building their position in the treatment of hematological malignancies and, in this article, we would like to overview the incidence of irAEs in this specific setting and also offer a brief insight into the management of irAEs. We took into account all available safety data from blood cancer ICI prospective clinical trials (both monotherapy and combination therapy) until April 2021 using Pubmed database and Google Scholar. The results are divided by affected organ systems.

\section{SKIN TOXICITY}

Dermatologic toxicity is the most common type of irAE in hematological malignancies. Maculopapular rash, dermatitis and pruritus represent the typical manifestation, but erythema nodosum, vitiligo, xerosis, psoriasis or even exfoliative dermatitis, skin necrosis and Stevens-Johnson syndrome were also observed (Badros et al., 2017; Khouri et al., 2018; Mateos M.-V. et al., 2019, Mateos et al., 2019 M. V.; Diefenbach et al., 2020; Song et al., 2020).

Rash and pruritus occur in $0-17 \%$ patients receiving ipilimumab (anti-CTLA-4 $\mathrm{mAb}$ ) in monotherapy and are mostly mild (grade 1-2). Interestingly, there was no skin toxicity attributed to ipilimumab administered in the postalogeneic stem cell transplant setting in the study conducted by Davids et al. (2016). Incidence was higher and irAEs slightly more severe in combination therapy, either with another ICI such as nivolumab (18-36\%) or with other immunotherapeutic agents (6-61\%) (Khouri et al., 2018; Diefenbach et al., 2020). Double immune checkpoint blockade even led to grade 4 StevensJohnson syndrome in one patient treated for cHL (Diefenbach et al., 2020).

Nivolumab (anti-PD-1 mAb) was associated with comparable allgrade skin toxicity in monotherapy (9-47\%) and in combination with other targeted therapeutics (13-31\%) (Armand et al., 2018a; Zinzani et al., 2019; Diefenbach et al., 2020; Maruyama et al., 2020). However, grade 3-4 cases were more common in the second cohort. Lower skin toxicity was observed in 2 combination studies of nivolumab and chemotherapy (AVD - doxorubicin, vinblastine, dacarbazine) for the treatment naïve $\mathrm{CHL}$, where only $0-6 \%$ of patients experienced skin irAEs (Ramchandren et al., 2019; Bröckelmann et al., 2020).

Another FDA-approved PD-1 inhibitor, pembrolizumab, caused dermatologic irAEs in $0-33 \%$ of patients (Khodadoust et al., 2016; Armand et al., 2019). In the CITN-10 study, it led to worsening of erythema and pruritus in patients with Sezary syndrome, but not in mycosis fungoides (Khodadoust et al., 2016). The flare reaction was associated with high expression of PD-1 on circulating Sézary cells, mostly remitted within 12 weeks with only symptomatic treatment and topical corticosteroids and did not lead to treatment discontinuation. 
TABLE 1 | Incidence of skin irAEs in hematological malignancies.

\begin{tabular}{|c|c|c|c|c|c|c|}
\hline \multicolumn{7}{|c|}{ Skin Toxicity } \\
\hline \multirow[t]{2}{*}{ Author } & \multirow[t]{2}{*}{ Condition } & \multirow[t]{2}{*}{ Phase } & \multirow[t]{2}{*}{ Regimen } & \multirow[t]{2}{*}{ Symptom } & \multicolumn{2}{|c|}{ Incidence } \\
\hline & & & & & all grade & grade $\geq 3$ \\
\hline \multirow[t]{2}{*}{ Armand et al. (2021) } & $\mathrm{RR}$ cHL, NHL, MM & $1 b$ & nivo + ipili & skin toxicity & $28 \%$ & NA \\
\hline & & & nivo + liri & & $24 \%$ & NA \\
\hline \multirow[t]{4}{*}{ Diefenbach et al. (2020) } & $\mathrm{RR} \mathrm{cHL}$ & $1 / 2$ & ipili + BV & pruritus/rash & $30 \% / 61 \%$ & $4 \% / 22 \%$ \\
\hline & & & nivo + BV & & $26 \% / 31 \%$ & $5 \% / 5 \%$ \\
\hline & & & ipili + nivo + BV & & $18 \% / 36 \%$ & $5 \% / 9 \%$ \\
\hline & & & ipili + nivo + BV & Stevens-Johnson sy & $5 \%$ & $5 \%$ \\
\hline Tuscano et al. (2019) & RR B-NHL & 1 & ipili + R & rash & $24 \%$ & $3 \%$ \\
\hline Khouri et al. (2018) & RR lymphoid malign. & 2 & ipili + lena & dermatitis & $6 \%$ & $0 \%$ \\
\hline Ansell et al. (2009) & RR B-NHL & 1 & ipili & pruritus/rash & $11 \% / 17 \%$ & 0\%/0\% \\
\hline Ribrag et al. (2021) & RR DLBCL & $1 b$ & durva + tremeli & dry skin & $33 \%$ & NA \\
\hline Maruyama et al. (2020) & $\mathrm{RR} \mathrm{cHL}$ & 2 & nivo & pruritus/rash & $35 \% / 47 \%$ & $0 \% / 6 \%$ \\
\hline Zinzani et al. (2019) & RR PMBL & $1 / 2$ & nivo + BV & rash & $13 \%$ & $3 \%$ \\
\hline Ramchandren et al. (2019) & $\mathrm{ND} \mathrm{cHL}$ & 2 & nivo + AVD & rash & $6 \%$ & $0 \%$ \\
\hline Younes et al. (2019) & RR NHL, RR CLL & $1 / 2 a$ & nivo + ibru & rash & NA & $8 \%$ \\
\hline Ansell et al. (2019) & RR DLBCL & 2 & nivo & rash & NA & $2 \%$ \\
\hline Armand et al. (2018a) & $\mathrm{RR} \mathrm{cHL}$ & 2 & nivo & rash & $9 \%$ & $2 \%$ \\
\hline Herrera et al. (2018) & $\mathrm{RR} \mathrm{cHL}$ & $1 / 2$ & nivo + BV & rash & NA & $2 \%$ \\
\hline Lesokhin et al. (2016) & RR hematol. malign. & $1 b$ & nivo & skin tox & $18 \%$ & $1 \%$ \\
\hline Ansell et al. (2015) & $\mathrm{RR} \mathrm{cHL}$ & 1 & nivo & pruritus/rash & $13 \% / 22 \%$ & $0 \% / 0 \%$ \\
\hline Khodadoust et al. (2016) & RR MF, RR SS & 2 & pembro & rash + skin flare & $42 \%$ & $17 \%$ \\
\hline Barta et al. (2019) & RR T-NHL & 2 & pembro & rash & $17 \%$ & $11 \%$ \\
\hline Armand et al. (2016) & $\mathrm{RR} \mathrm{cHL}$ & $1 b$ & pembro & xerosis & $2 \%$ & $0 \%$ \\
\hline Liu et al. (2021) & $\mathrm{RR} \mathrm{cHL}$ & 2 & camre + decitabine & rash & $12 \%$ & $0 \%$ \\
\hline Mei et al. (2020) & $\mathrm{RR} P M B L$ & 2 & camre + GVD & pruritus/rash & $30 \% / 22 \%$ & 0\%/0\% \\
\hline Kazandjian et al. (2021) & RR MM & 2 & avelumab & rash & $25 \%$ & $0 \%$ \\
\hline D'Souza et al. (2019) & ND MM & 2 & pembro + lena & rash & $10 \%$ & $0 \%$ \\
\hline Usmani et al. (2019) & ND MM & 3 & pembro + lena + dex & rash & $9 \%$ & $9 \%$ \\
\hline Mateos et al. (2019a) & $\mathrm{RR} M \mathrm{MM}$ & 1 & pembro + lena + dex & exfoliative dermatitis & $2 \%$ & $0 \%$ \\
\hline Ribrag et al. (2019) & RR MM & $1 b$ & pembro & pruritus & $3 \%$ & $0 \%$ \\
\hline Mateos et al. (2019b) & RR MM & 3 & pembro + pom + dex & rash & $3 \%$ & $3 \%$ \\
\hline & & & & Stevens-Johnson sy & $1 \%$ & $1 \%$ \\
\hline Badros et al. (2017) & $\mathrm{RR} \mathrm{MM}$ & 2 & pembro + pom + dex & vitiligo & $2 \%$ & $0 \%$ \\
\hline Zeidan et al. (2018) & RR MDS & $1 b$ & ipili & dermatitis + rash & $14 \%$ & $3 \%$ \\
\hline Davids et al. (2020) & RR hematol. malign. & 1 & nivo & rash & $29 \%$ & $4 \%$ \\
\hline Ravandi et al. (2019) & ND AML, ND MDS & 2 & nivo + ida + cytarabine & rash & NA & $5 \%$ \\
\hline Cassaday et al. (2020) & ND/RR ALL & 2 & pembro & Stevens-Johnson sy & $8 \%$ & $8 \%$ \\
\hline
\end{tabular}

RR, relapsed/refractory; ND, newly diagnosed; CHL, classical Hodgkin lymphoma; NHL, non-Hodgkin lymphoma; MM, multiple myeloma; PMBL, primary mediastinal large B cell lymphoma; CLL, chronic lymphocytic leukemia; DLBCL, diffuse large B cell lymphoma; FL, follicular lymphoma; MF, mycosis fungoides; SS, Sézary syndrome; AML, acute myeloid leukemia; MDS, myelodysplastic syndrome; ALL, acute lymphoblastic leukemia; ipili, ipilimumab; nivo, nivolumab; BV, brentuximab vedotin; liri, lirilumab; R, rituximab; AVD, adriamycin + vinblastine + dacarbazine; GVD, gemcitabine + vinorelbine + doxorubicin; camre, camrelizumab; pembro, pembrolizumab; ibru, ibrutinib; lena, lenalidomide; pom, pomalidomide; dex, dexamethasone; ida, idarubicin; durva, durvalumab; tremeli, tremelimumab; NA, not available.

Pembrolizumab combined with immunomodulatory drugs (IMiDs) and dexamethasone was extensively investigated in multiple myeloma. Skin related toxicity was not very frequent (2-10\%), but could be considered more severe, because one patient died of treatment-related grade 5 Stevens-Johnson syndrome (D’Souza et al., 2019; Mateos M. V. et al., 2019, Mateos et al., 2019 M.-V.). Interestingly, one myeloma patient developed vitiligo on ICI treatment, which is almost exclusively seen in melanomas (Badros et al., 2017; Ransohoff and Kwong, 2017; Chhabra and Kennedy, 2021).

Anti-PD-L1 mAbs are not yet so widely investigated as antiPD-1 mAbs, but the skin toxicity seems comparable with rash and dry skin reported from available clinical trial data (Kazandjian et al., 2021; Ribrag et al., 2021). (Table 1)
Grade 1 (affecting $<10 \%$ of body surface area $=$ BSA) and grade 2 (10-30\% BSA) dermatologic irAEs are typically managed with topical emollients, topical steroids and/or antihistamines, avoiding skin irritants or sun exposure and usually do not lead to ICI treatment discontinuation (Haanen et al., 2017; Chhabra and Kennedy, 2021). More severe cases are associated with affection of $>30 \%$ BSA and cooperation with a dermatology specialist and punch biopsy is recommended. Grade 3 cases are managed with oral (prednisolone $0.5-1 \mathrm{mg} / \mathrm{kg}$ ) or intravenous (methylprednisolone $0.5-1 \mathrm{mg} / \mathrm{kg}$ ) systemic steroids and, after resolving the symptoms to grade 1 or mild grade 2 , ICIs can be restarted after thorough risk/benefit assessment and discussion with the patient. Steroids are recommended to be weaned over 1-2 and 2-4 weeks respectively, because of the risk of relapse. Grade 4 is a 
TABLE 2 | Incidence of endocrine irAEs in hematological malignancies.

\begin{tabular}{|c|c|c|c|c|c|c|}
\hline \multicolumn{7}{|c|}{ Endocrine Toxicity } \\
\hline \multirow[t]{2}{*}{ Author } & \multirow[t]{2}{*}{ Condition } & \multirow[t]{2}{*}{ Phase } & \multirow[t]{2}{*}{ Regimen } & \multirow[t]{2}{*}{ Symptom } & \multicolumn{2}{|c|}{ Incidence } \\
\hline & & & & & all grade & grade $\geq 3$ \\
\hline Diefenbach et al. (2020) & $\mathrm{RR} \mathrm{cHL}$ & $1 / 2$ & ipili + nivo + BV & endocrine disorders & $5 \%$ & $5 \%$ \\
\hline \multirow[t]{2}{*}{ Khouri et al. (2018) } & RR Iymphoid malign. & 2 & ipili + lena & hypothyroidism & $6 \%$ & $0 \%$ \\
\hline & & & & hyperthyroidism & $6 \%$ & $0 \%$ \\
\hline Bashey et al. (2009) & cancer post HSCT & 1 & ipili & hyperthyroidism & $3 \%$ & $0 \%$ \\
\hline Ansell et al. (2009) & RR B-NHL & 1 & ipili & hypophysitis susp. & $8 \%$ & $0 \%$ \\
\hline \multirow[t]{2}{*}{ Maruyama et al. (2020) } & $\mathrm{RR} \mathrm{cHL}$ & 2 & nivo & hypothyroidism & $29 \%$ & $0 \%$ \\
\hline & & & & fulminant DM 1T & $6 \%$ & $6 \%$ \\
\hline Bröckelmann et al. (2020) & $\mathrm{ND} \mathrm{cHL}$ & 2 & nivo + AVD & hypothyroidism & $7 \%$ & $0 \%$ \\
\hline \multirow[t]{3}{*}{ Zinzani et al. (2019) } & RR PMBL & $1 / 2$ & nivo + BV & hyperthyroidism & $13 \%$ & $0 \%$ \\
\hline & & & & hypothyroidism & $7 \%$ & $0 \%$ \\
\hline & & & & thyroiditis & $3 \%$ & $0 \%$ \\
\hline \multirow[t]{2}{*}{ Ramchandren et al. (2019) } & $\mathrm{ND} \mathrm{cHL}$ & 2 & nivo + AVD & hyperthyroidism & $8 \%$ & $0 \%$ \\
\hline & & & & hypothyroidism/thyroiditis & $18 \%$ & $0 \%$ \\
\hline \multirow[t]{4}{*}{ Armand et al. (2018a) } & $\mathrm{RR} \mathrm{cHL}$ & 2 & nivo & hyperthyroidism & $2 \%$ & $0 \%$ \\
\hline & & & & hypothyroidism/thyroiditis & $12 \%$ & $0 \%$ \\
\hline & & & & $\mathrm{DM}$ & $1 \%$ & $1 \%$ \\
\hline & & & & adrenal insufficiency & $1 \%$ & $0 \%$ \\
\hline Ansell et al. (2015) & $\mathrm{RR} \mathrm{cHL}$ & 1 & nivo & hypothyroidism & $9 \%$ & $0 \%$ \\
\hline \multirow[t]{4}{*}{ Armand et al. (2019) } & RR PMBL & $1 b$ & pembro & hypothyroidism & $9 \%$ & $0 \%$ \\
\hline & & 2 & & hypothyroidism & $8 \%$ & $0 \%$ \\
\hline & & & & hyperthyroidism & $4 \%$ & $0 \%$ \\
\hline & & & & thyroiditis & $2 \%$ & $0 \%$ \\
\hline \multirow[t]{2}{*}{ Barta et al. (2019) } & RR T-NHL & 2 & pembro & hypothyroidism & $11 \%$ & $11 \%$ \\
\hline & & & & adrenal insufficiency & $6 \%$ & NA \\
\hline \multirow[t]{2}{*}{ Armand et al. (2016) } & $\mathrm{RR} \mathrm{cHL}$ & $1 b$ & pembro & hypothyroidism & $16 \%$ & $0 \%$ \\
\hline & & & & thyroiditis & $6 \%$ & $0 \%$ \\
\hline \multirow[t]{3}{*}{ Liu et al. (2021) } & $\mathrm{RR} \mathrm{cHL}$ & 2 & camre & hypothyroidism & $16 \%$ & $0 \%$ \\
\hline & & & camre + decitabine & hypothyroidism & $7 \%$ & $0 \%$ \\
\hline & & & & hypoparathyroidism & $2 \%$ & $0 \%$ \\
\hline \multirow[t]{2}{*}{ D'Souza et al. (2019) } & ND MM & 2 & pembro + lena & hypothyroidism & $17 \%$ & $0 \%$ \\
\hline & & & & hyperthyroidism & $17 \%$ & $0 \%$ \\
\hline \multirow[t]{2}{*}{ Usmani et al. (2019) } & ND MM & 3 & pembro + lena + dex & hypothyroidism & $7 \%$ & $0 \%$ \\
\hline & & & & hyperthyroidism & $6 \%$ & $2 \%$ \\
\hline \multirow[t]{2}{*}{ Mateos et al. (2019a) } & $\mathrm{RR} M \mathrm{MM}$ & 1 & pembro + lena + dex & hypothyroidism & $8 \%$ & $0 \%$ \\
\hline & & & & hyperthyroidism & $6 \%$ & $0 \%$ \\
\hline Ribrag et al. (2019) & RR MM & $1 b$ & pembro & hypothyroidism & $3 \%$ & $0 \%$ \\
\hline \multirow[t]{2}{*}{ Mateos et al. (2019b) } & $\mathrm{RR} \mathrm{MM}$ & 3 & pembro + pom + dex & hyperthyroidism & $3 \%$ & $0 \%$ \\
\hline & & & & hypothyroidism & $2 \%$ & $0 \%$ \\
\hline \multirow[t]{2}{*}{ Badros et al. (2017) } & $\mathrm{RR} \mathrm{MM}$ & 2 & pembro + pom + dex & hypothyroidism & $10 \%$ & $4 \%$ \\
\hline & & & & adrenal insufficiency & $4 \%$ & $2 \%$ \\
\hline
\end{tabular}

RR, relapsed/refractory; ND, newly diagnosed; CHL, classical Hodgkin lymphoma; NHL, non-Hodgkin lymphoma; MM, multiple myeloma; PMBL, primary mediastinal large B cell lymphoma; CLL, chronic lymphocytic leukemia; DLBCL, diffuse large B cell lymphoma; FL, follicular lymphoma; HSCT, (allogeneic) hematopoietic stem cell transplantation; ipili, ipilimumab; nivo, nivolumab; BV, brentuximab vedotin; AVD, adriamycin + vinblastine + dacarbazine; camre, camrelizumab; pembro; pembrolizumab; lena, lenalidomide; pom, pomalidomide; dex, dexamethasone; susp., suspect; DM, diabetes mellitus; DM 1T, type 1 diabetes mellitus; NA, not available.

life-threatening situation associated with symptoms such as erythema, purpura or epidermal detachment, requires rapid administration of intravenous steroids (methylprednisolone $1-2 \mathrm{mg} / \mathrm{kg}$ ) and permanent discontinuation of ICI treatment.

\section{ENDOCRINE TOXICITY}

Patients receiving ICI treatment relatively often experience irAEs affecting endocrine organs - mostly the thyroid gland, pituitary gland, adrenal glands and pancreas (Chang et al., 2019; Chhabra and Kennedy, 2021). Anti-CTLA-4 mAbs are connected with higher rates of hypophysitis, whereas PD-1 inhibitors more frequently induce hypothyroidism, hyperthyroidism or thyroiditis. Type 1 diabetes mellitus and adrenal insufficiency are less common.

Ipilimumab use in hematological malignancies, both in monotherapy or combination therapy, was associated with low incidence of thyroid dysfunction (0-6\%) (Davids et al., 2016; Khouri et al., 2018). Only one case of suspected mild hypophysitis was reported in a phase 1 study for RR B-NHL (Ansell et al., 2009). This patient with a history of type 2 diabetes mellitus developed hyperglycemia during upper respiratory tract infection, anorexia, anemia, weight loss and erectile 
dysfunction. Laboratory findings uncovered low serum testosterone, low-normal gonadotropin levels and a normal MRI pituitary scan.

Both FDA-approved PD-1 inhibitors, nivolumab and pembrolizumab, showed similar thyroid toxicity. Hypothyroidism occurred in $0-29 \%$ and $0-17 \%$ of cases, respectively, while hyperthyroidism was reported in $0-13 \%$ and $0-17 \%$ of cases, respectively (Khodadoust et al., 2016; D'Souza et al., 2019; Younes et al., 2019; Zinzani et al., 2019; Maruyama et al., 2020). Adrenal insufficiency was also observed in both of these agents with the maximum incidence of 6\% (Badros et al., 2017; Armand et al., 2018a; Barta et al., 2019; Ribrag et al., 2019). Type 1 diabetes mellitus was associated with nivolumab use in two RR cHL clinical trials (1 patient each) (Armand et al., 2018a; Maruyama et al., 2020). The rare endocrine irAE, hypoparathyroidism, affected one patient with $\mathrm{cHL}$ while receiving camrelizumab (anti-PD-1 $\mathrm{mAb}$ ) and decitabine (hypomethylating agent) combination treatment (Liu et al., 2021). There was no endocrine toxicity observed in anti-PDL1 based hematological clinical trials and in all available studies investigating ICI use in acute leukemia or myelodysplastic syndrome (Ravandi et al., 2019; Cassaday et al., 2020; Kazandjian et al., 2021; Ribrag et al., 2021). (Table 2)

Endocrine disorders during ICI therapy are usually mild to moderate (grade 1 and 2), but in a minority of cases, can be severe or life-threatening (Haanen et al., 2017; Chang et al., 2019; Chhabra and Kennedy, 2021). Thyroid-targeting irAEs are seen in up to $80 \%$ of cases caused by anti-thyroid antibodies. Hyperthyroidism is often transient and within 3-6 weeks usually leads to permanent hypothyroidism which does not resolve after ICI discontinuation and requires lifelong thyroid hormone replacement. It is recommended to perform thyroid function tests $(\mathrm{TSH}=$ thyroid-stimulating hormone, $\mathrm{fT} 4=$ free thyroxine, $\mathrm{fT} 3=$ free tri-iodothyronine) every cycle for the first 3 months of anti-PD-(L) 1 treatment and every second cycle thereafter. Cortisol level should be taken when TSH is falling and/or symptoms suggesting secondary (central) hypothyroidism occur. It can be a sign of hypopituitarism and potentially life-threatening central adrenal insufficiency when rapid corticosteroid replacement prevents development of adrenal crisis. In the rare cases of symptomatic thyreotoxicosis, beta-blockers (propranolol or atenolol) are recommended. Antithyroid drugs are not routinely used unless Graves' disease is diagnosed. In the case of painful thyroiditis, prednisolone in the dosage of $0.5 \mathrm{mg} / \mathrm{kg}$ should be considered. Thyroid disorders usually do not lead to ICI discontinuation.

Blood glucose levels should be monitored regularly in patients treated with ICIs because of the danger of de novo type 1 diabetes mellitus (Haanen et al., 2017; Chang et al., 2019). Patients with pre-existing type 2 diabetes mellitus can develop diabetic ketoacidosis. These complications should be managed according to local practice; high-dose steroids are not recommended. Affected patients can restart ICI treatment after they are stable and insulin therapy is adjusted. ICI discontinuation does not lead to the restitution of the endogenous insulin level.

Primary adrenal insufficiency (PAI) differs from abovementioned central adrenal insufficiency by elevated ACTH (adrenocorticotropic hormone) and low cortisol level (Chang et al., 2019). The difference is also in management, as PAI requires rapid mineralocorticoid replacement in addition to glucocorticoids.

\section{HEPATOTOXICITY}

ICI related liver injury shares many features with autoimmune hepatitis. In most cases, it presents with asymptomatic elevation of alanine aminotransferase (ALT), aspartate aminotransferase (AST) or total bilirubin, but severe hepatitis and liver failure associated with fever, malaise, jaundice and change of stool color can also occur (Tian et al., 2018; Chhabra and Kennedy, 2021).

The incidence of abnormal ALT and AST levels is comparable between CTLA-4 and PD-1 inhibitors. The most prominent treatment-related hepatotoxicity was observed in a phase $1 / 2$ clinical trial investigating the efficacy of ipilimumab, nivolumab and brentuximab vedotin (BV) in RR cHL patients - the rate of ALT/AST increase was as high as $48 \% / 39 \%$ for the ipilimumab + BV cohort and $47 \% / 32 \%$ for the nivolumab + BV cohort (Diefenbach et al., 2020). All of these events were grade 1 to 2 in severity. Singular grade 3 cases were detected in the triple therapy group. Slightly less toxic anti-PD-1 mAbs appeared to be pembrolizumab and camrelizumab with only $0-8 \%$ and $0 \%$ proportion of patients experiencing liver dysfunction, respectively (Khodadoust et al., 2016; Armand et al., 2019; Mei et al., 2020; Liu et al., 2021). Hepatotoxicity was also reported in anti-PD-L1 hemato-oncological trials, but the cohorts were small, so the results should be regarded with caution (Kazandjian et al., 2021; Ribrag et al., 2021). (Table 3)

When considering hepatic irAE, other causes of liver injury such as medication, autoimmunity, viral infection and alcohol should be ruled out in the first place (Haanen et al., 2017; Tian et al., 2018; Chhabra and Kennedy, 2021). Imaging methods (CT = computer tomography, $\mathrm{MRI}=$ magnetic resonance imaging, Doppler ultrasound) or liver biopsy could help in disputable situations. Management is dependent on severity of damage. Grade 1 (1-3x ULN = upper limit of normal) cases are closely monitored at least once weekly and ICI treatment can continue. When ALT/AST levels reach grade 2 (3-5x ULN), ICI should be temporary withheld and oral corticosteroid therapy initiated (prednisolone $1 \mathrm{mg} / \mathrm{kg}$ ). Grade 3 and 4 liver injuries (5-20x ULN and >20 ULN, respectively) lead to permanent ICI discontinuation and systemic steroid treatment. In grade 3 cases with ALT/ AST $<400$, normal bilirubin, INR (International Normalized Ratio) and albumin oral prednisolone $1 \mathrm{mg} / \mathrm{kg}$ is suggested. Higher dosage $(2 \mathrm{mg} / \mathrm{kg})$ of prednisolone should be administered when there is ALT/AST $>400$, elevated bilirubin or INR or decreased albumin. Grade 4 toxicity is managed by (methyl)prednisolone $2 \mathrm{mg} / \mathrm{kg}$. Steroid- 
TABLE 3 | Incidence of hepatic irAEs in hematological malignancies.

\begin{tabular}{|c|c|c|c|c|c|c|}
\hline \multicolumn{7}{|c|}{ Hepatotoxicity } \\
\hline \multirow[t]{2}{*}{ Author } & \multirow[t]{2}{*}{ Condition } & \multirow[t]{2}{*}{ Phase } & \multirow[t]{2}{*}{ Regimen } & \multirow[t]{2}{*}{ Symptom } & \multicolumn{2}{|c|}{ Incidence } \\
\hline & & & & & all grade & grade $\geq \mathbf{3}$ \\
\hline Armand et al. (2021) & $\mathrm{RR} \mathrm{cHL}, \mathrm{NHL}, \mathrm{MM}$ & $1 b$ & nivo + ipili & elevated ALT/AST & NA & $3 \% / 2 \%$ \\
\hline \multirow[t]{3}{*}{ Diefenbach et al. (2020) } & $\mathrm{RR} \mathrm{cHL}$ & $1 / 2$ & ipili + BV & elevated ALT/AST & $48 \% / 39 \%$ & 0\%/0\% \\
\hline & & & nivo + BV & & $47 \% / 32 \%$ & $0 \% / 0 \%$ \\
\hline & & & ipili + nivo + BV & & $14 \% / 14 \%$ & $5 \% / 5 \%$ \\
\hline Ansell et al. (2009) & RR B-NHL & 1 & ipili & elevated AST & $22 \%$ & $0 \%$ \\
\hline Maruyama et al. (2020) & $\mathrm{RR} \mathrm{cHL}$ & 2 & nivo & abnormal hepatic function & $12 \%$ & $6 \%$ \\
\hline Zinzani et al. (2019) & RR PMBL & $1 / 2$ & nivo + BV & hepatitis & $3 \%$ & $3 \%$ \\
\hline \multirow[t]{2}{*}{ Ramchandren et al. (2019) } & $\mathrm{ND} \mathrm{cHL}$ & 2 & nivo + AVD & hepatitis & $4 \%$ & $4 \%$ \\
\hline & & & & elevated ALT/AST & $4 \% / 2 \%$ & $4 \% / 2 \%$ \\
\hline Younes et al. (2019) & RR NHL, RR CLL & $1 / 2 a$ & nivo + ibru & elevated ALT/AST & NA/NA & $2 \% / 1 \%$ \\
\hline Ansell et al. (2019) & RR DLBCL & 2 & nivo & abnormal hepatic function & NA & $3 \%$ \\
\hline Armand et al. (2018a) & $\mathrm{RR} \mathrm{cHL}$ & 2 & nivo & hepatitis & $5 \%$ & $4 \%$ \\
\hline Herrera et al., 2018 & $\mathrm{RR} \mathrm{cHL}$ & $1 / 2$ & nivo + BV & elevated ALT/AST & NA/NA & $2 \% / 2 \%$ \\
\hline Lesokhin et al. (2016) & RR hematol. malign. & $1 b$ & nivo & elevated ALT + AST & $2 \%$ & $0 \%$ \\
\hline Khodadoust et al. (2016) & RR MF, RR SS & 2 & pembro & elevated ALT/AST & $4 \% / 8 \%$ & $4 \% / 4 \%$ \\
\hline Barta et al. (2019) & RR T-NHL & 2 & pembro & abnormal hepatic function & $6 \%$ & $0 \%$ \\
\hline Armand et al. (2016) & $\mathrm{RR} \mathrm{cHL}$ & $1 b$ & pembro & elevated ALT/AST & $6 \% / 6 \%$ & $3 \% / 3 \%$ \\
\hline Ribrag et al. (2021) & RR DLBCL & $1 b$ & durva + danvatirsen & elevated ALT/AST & $33 \% / 29 \%$ & NA/NA \\
\hline Kazandjian et al. (2021) & $\mathrm{RR} \mathrm{MM}$ & 2 & avelumab & elevated ALT/AST & $25 \% / 25 \%$ & 0\%/0\% \\
\hline D’Souza et al. (2019) & ND MM & 2 & pembro + lena & hepatitis & $3 \%$ & $3 \%$ \\
\hline \multirow[t]{2}{*}{ Usmani et al. (2019) } & ND MM & 3 & pembro + lena + dex & hepatitis & $1 \%$ & $1 \%$ \\
\hline & & & & drug-induced liver injury & $1 \%$ & $1 \%$ \\
\hline Mateos et al. (2019a) & RR MM & 3 & pembro + pom + dex & hepatitis & $1 \%$ & $1 \%$ \\
\hline Badros et al. (2017) & $\mathrm{RR} \mathrm{MM}$ & 2 & pembro + pom + dex & hepatitis & $4 \%$ & $2 \%$ \\
\hline Zeidan et al. (2018) & RR MDS & $1 b$ & ipili & transaminitis & $7 \%$ & $7 \%$ \\
\hline Davids et al. (2020) & RR hematol. malign. & 1 & nivo & transaminitis & $29 \%$ & $7 \%$ \\
\hline Ravandi et al. (2019) & ND AML, ND MDS & 2 & nivo + ida + cytarabine & transaminitis & NA & $3 \%$ \\
\hline Daver et al. (2019) & RR AML & 2 & nivo + azacitidine & transaminitis & NA & $3 \%$ \\
\hline
\end{tabular}

RR, relapsed/refractory; ND, newly diagnosed; CHL, classical Hodgkin lymphoma; NHL, non-Hodgkin lymphoma; MM, multiple myeloma; PMBL, primary mediastinal large B cell lymphoma; CLL, chronic lymphocytic leukemia; DLBCL, diffuse large B cell lymphoma; FL, follicular lymphoma; MF, mycosis fungoides; SS, Sézary syndrome; AML, acute myeloid leukemia; MDS, myelodysplastic syndrome; ALL, acute lymphoblastic leukemia; ipili, ipilimumab; nivo, nivolumab; BV, brentuximab vedotin; AVD, adriamycin + vinblastine + dacarbazine; pembro, pembrolizumab; lena, lenalidomide; pom, pomalidomide; dex, dexamethasone; ibru, ibrutinib; ida, idarubucin; durva, durvalumab; ALT, alanine aminotransferase; AST, aspartate aminotransferase ; NA, not available.

refractory patients might benefit from mycophenolate mofetil (500-1000 mg twice a day), tacrolimus or anti-thymocyte globulin. Infliximab is not recommended due to its potential hepatotoxic effect.

\section{GASTROINTESTINAL TOXICITY}

Gastrointestinal (GI) irAEs occur frequently, especially during anti-CTLA-4 mAb treatment (Rocha et al., 2019; Chhabra and Kennedy, 2021). Their clinical presentation can be variable with diarrhoea, abdominal pain, hematochezia, weight loss, fever, vomiting, mouth ulcers, anal lesions and even extraintestinal manifestations reported (Haanen et al., 2017). Upper GI symptoms (nausea, vomiting, dysphagia, epigastric pain) are less common (Haanen et al., 2017; Rocha et al., 2019).

In hemato-oncological clinical trials, ipilimumab showed similar GI toxicity as in solid tumors. Diarrhoea was the most usual manifestation, observed in a maximum of 56 and $61 \%$ patients receiving ipilimumab monotherapy or combination therapy, respectively (Ansell et al., 2009; Diefenbach et al., 2020). Colitis was reported in a maximum of 7 and 5\%, respectively (Zeidan et al., 2018; Diefenbach et al., 2020). Double immune checkpoint blockade (ipilimumab + nivolumab) was associated also with gastritis and a severe case of autoimmune pancreatitis (Diefenbach et al., 2020; Armand et al., 2021).

Anti-PD-1 and anti-PD-L1 agents seemed to be less toxic to the GI tract (Chhabra and Kennedy, 2021). Diarrhoea was also the most commonly reported GI symptom, but the incidence did not exceed 33\% (Ribrag et al., 2021). Other types of manifestation included colitis, typhlitis, enteritis, proctitis, proctosigmoiditis, pancreatitis, cholecystitis and duodenitis (Khodadoust et al., 2016; Armand et al., 2019; Ravandi et al., 2019; Diefenbach et al., 2020; Maruyama et al., 2020). No grade 5 GI ICI-related toxicity has been reported so far. (Table 4)

When considering GI toxicity of ICIs, bacterial or viral infection should be excluded at first (Haanen et al., 2017; Rocha et al., 2019; Chhabra and Kennedy, 2021). GI involvement associated with the main diagnosis is not as frequently seen in hematological malignancies as in solid 
TABLE 4 | Incidence of gastrointestinal irAEs in hematological malignancies.

\begin{tabular}{|c|c|c|c|c|c|c|}
\hline \multicolumn{7}{|c|}{ Gastrointestinal Toxicity } \\
\hline \multirow[t]{2}{*}{ Author } & \multirow[t]{2}{*}{ Condition } & \multirow[t]{2}{*}{ Phase } & \multirow[t]{2}{*}{ Regimen } & \multirow[t]{2}{*}{ Symptom } & \multicolumn{2}{|c|}{ Incidence } \\
\hline & & & & & all grade & grade $\geq \mathbf{3}$ \\
\hline \multirow[t]{4}{*}{ Armand et al. (2021) } & $\mathrm{RR} \mathrm{cHL}, \mathrm{NHL}, \mathrm{MM}$ & $1 b$ & nivo + ipili & diarrhoea & $19 \%$ & $2 \%$ \\
\hline & & & & colitis & $2 \%$ & $2 \%$ \\
\hline & & & & Al pancreatitis & $2 \%$ & $2 \%$ \\
\hline & & & nivo + liri & diarrhoea & $11 \%$ & $0 \%$ \\
\hline \multirow[t]{6}{*}{ Diefenbach et al. (2020) } & $\mathrm{RR} \mathrm{cHL}$ & $1 / 2$ & ipili + BV & diarrhoea & $61 \%$ & $4 \%$ \\
\hline & & & nivo + BV & diarrhoea & $21 \%$ & ०\% \\
\hline & & & & typhlitis & $5 \%$ & $5 \%$ \\
\hline & & & ipili + nivo + BV & diarrhoea & $45 \%$ & $5 \%$ \\
\hline & & & & colitis & $5 \%$ & $5 \%$ \\
\hline & & & & gastritis & $10 \%$ & $5 \%$ \\
\hline \multirow[t]{2}{*}{ Tuscano et al. (2019) } & RR B-NHL & 1 & ipili + R & diarrhoea & $30 \%$ & $12 \%$ \\
\hline & & & & colitis & $3 \%$ & $3 \%$ \\
\hline \multirow[t]{2}{*}{ Davids et al. (2016) } & RR hematol. malign. & $1 / 1 b$ & ipili & diarrhoea & $4 \%$ & $0 \%$ \\
\hline & & & & colitis & $4 \%$ & $4 \%$ \\
\hline Ansell et al. (2009) & RR B-NHL & 1 & ipili & diarrhoea & $56 \%$ & $28 \%$ \\
\hline \multirow[t]{2}{*}{ Maruyama et al. (2020) } & $\mathrm{RR} \mathrm{cHL}$ & 2 & nivo & diarrhoea & $6 \%$ & $0 \%$ \\
\hline & & & & enteritis/proctitis & $6 \% / 12 \%$ & $0 \% / 6 \%$ \\
\hline Bröckelmann et al. (2020) & $\mathrm{ND} \mathrm{cHL}$ & 2 & nivo + AVD & proctosigmoiditis & $1 \%$ & $1 \%$ \\
\hline Ramchandren et al. (2019) & $\mathrm{ND} \mathrm{cHL}$ & 2 & nivo + AVD & diarrhoea & $10 \%$ & $0 \%$ \\
\hline Zinzani et al. (2019) & RR PMBL & $1 / 2$ & nivo + BV & colitis & $3 \%$ & $3 \%$ \\
\hline \multirow[t]{2}{*}{ Younes et al. (2019) } & RR NHL, RR CLL & $1 / 2 a$ & nivo + ibru & diarrhoea & NA & $1 \%$ \\
\hline & & & & colitis/enterocolitis & NA & $1 \% / 1 \%$ \\
\hline Ansell et al. (2019) & RR DLBCL & 2 & nivo & diarrhoea & NA & $3 \%$ \\
\hline \multirow[t]{2}{*}{ Armand et al. (2018a) } & $\mathrm{RR} \mathrm{cHL}$ & 2 & nivo & diarrhoea & $2 \%$ & $1 \%$ \\
\hline & & & & colitis & $2 \%$ & $1 \%$ \\
\hline \multirow[t]{2}{*}{ Herrera et al., 2018} & $\mathrm{RR} \mathrm{cHL}$ & $1 / 2$ & nivo + BV & diarrhoea & NA & $3 \%$ \\
\hline & & & & colitis & NA & $3 \%$ \\
\hline Lesokhin et al. (2016) & RR hematol. malign. & $1 b$ & nivo & diarrhoea + enteritis & $7 \%$ & $0 \%$ \\
\hline \multirow[t]{2}{*}{ Ansell et al. (2015) } & $\mathrm{RR} \mathrm{cHL}$ & 1 & nivo & diarrhoea & $13 \%$ & $0 \%$ \\
\hline & & & & pancreatitis & $4 \%$ & $4 \%$ \\
\hline Khodadoust et al. (2016) & RR MF, RR SS & 2 & pembro & colitis + duodenitis & $8 \%$ & $4 \%$ \\
\hline Barta et al. (2019) & RR T-NHL & 2 & pembro & diarrhoea & $6 \%$ & ०\% \\
\hline Armand et al. (2019) & RR PMBL & $1 b$ & pembro & colitis & $5 \%$ & $0 \%$ \\
\hline Armand et al. (2016) & $\mathrm{RR} \mathrm{cHL}$ & $1 b$ & pembro & diarrhoea/colitis & $16 \% / \mathrm{NA}$ & NA/3\% \\
\hline Liu et al. (2021) & $\mathrm{RR} \mathrm{cHL}$ & 2 & camre + decitabine & diarrhoea & $10 \%$ & $0 \%$ \\
\hline Ribrag et al. (2021) & RR DLBCL & $1 b$ & durva + tremeli & diarrhoea & $33 \%$ & NA \\
\hline D'Souza et al. (2019) & ND MM & 2 & pembro + lena & colitis & $13 \%$ & $3 \%$ \\
\hline Usmani et al. (2019) & ND MM & 3 & pembro + lena + dex & colitis/pancreatitis & $2 \% / 1 \%$ & $1 \% / 1 \%$ \\
\hline Zeidan et al. (2018) & RR MDS & $1 b$ & ipili & colitis & $7 \%$ & $3 \%$ \\
\hline Davids et al. (2020) & RR hematol. malign. & 1 & nivo & diarrhoea & $11 \%$ & $0 \%$ \\
\hline \multirow[t]{2}{*}{ Ravandi et al. (2019) } & ND AML, ND MDS & 2 & nivo + ida + cytarabine & colitis/pancreatitis & NA/NA & $5 \% / 3 \%$ \\
\hline & & & & cholecystitis & NA & $3 \%$ \\
\hline Daver et al. (2019) & RR AML & 2 & nivo + azacitidine & colitis/enterocolitis & $1 \% / 1 \%$ & $1 \% / 0 \%$ \\
\hline
\end{tabular}

RR, relapsed/refractory; ND, newly diagnosed; CHL, classical Hodgkin lymphoma; NHL, non-Hodgkin lymphoma; MM, multiple myeloma; PMBL, primary mediastinal large B cell lymphoma; CLL, chronic lymphocytic leukemia; DLBCL, diffuse large B cell lymphoma; MF, mycosis fungoides; SS, Sézary syndrome; AML, acute myeloid leukemia; MDS, myelodysplastic syndrome; ipili, ipilimumab; liri, lirilumab; nivo, nivolumab; BV, brentuximab vedotin; AVD, adriamycin + vinblastine + dacarbazine; R, rituximab; pembro, pembrolizumab; lena, lenalidomide; dex, dexamethasone; ibru, ibrutinib; ida, idarubucin; durva, durvalumab; tremeli, tremelimumab; camre, camrelizumab; Al, autoimmune; NA, not available.

oncology, when metastases could cause symptoms similar to GI irAEs. Laboratory tests, abdominal $\mathrm{X}$ ray or $\mathrm{CT}$ scan and endoscopic methods could help in dubious cases. The benefit of biopsy should be carefully assessed due to the risk of perforation. GI irAEs may mimic idiopathic inflammatory bowel disease.

Grade 1 colitis is defined by $<4$ liquid stools per day and the treatment is symptomatic with mainly fluid and electrolyte repletion (Haanen et al., 2017; Chhabra and Kennedy, 2021).
When grade 1 colitis persists $>14$ days or grade 2 (4-6 liquid stools) persists $>3$ days, ICI treatment should be withheld and oral steroids (prednisolone $0.5-1 \mathrm{mg} / \mathrm{kg}$ or budesonide $9 \mathrm{mg}$ once daily) immediately initiated. If no improvement is seen after $72 \mathrm{~h}$ or in grade 3-4 cases ( $\geq 7$ liquid stools per day), intravenous (methyl)prednisolone $1-2 \mathrm{mg} / \mathrm{kg}$ is recommended with possible escalation to infliximab $5 \mathrm{mg} / \mathrm{kg}$ after another $72 \mathrm{~h}$. Other possible immunosuppressive treatment options include mycophenolate mofetil (500-1000 mg twice a day) or 
TABLE 5 | Incidence of pulmonary irAEs in hematological malignancies.

\begin{tabular}{|c|c|c|c|c|c|c|}
\hline \multicolumn{7}{|c|}{ Pneumotoxicity } \\
\hline \multirow[t]{2}{*}{ Author } & \multirow[t]{2}{*}{ Condition } & \multirow[t]{2}{*}{ Phase } & \multirow[t]{2}{*}{ Regimen } & \multirow[t]{2}{*}{ Symptom } & \multicolumn{2}{|c|}{ Incidence } \\
\hline & & & & & all grade & grade $\geq 3$ \\
\hline \multirow[t]{2}{*}{ Armand et al. (2021) } & $\mathrm{RR} \mathrm{cHL}, \mathrm{NHL}, \mathrm{MM}$ & $1 b$ & nivo + ipili & pneumonitis & $12 \%$ & $5 \%$ \\
\hline & & & nivo + liri & & $1 \%$ & $0 \%$ \\
\hline \multirow[t]{3}{*}{ Diefenbach et al. (2020) } & $\mathrm{RR} \mathrm{cHL}$ & $1 / 2$ & ipili + BV & pneumonitis & $4 \%$ & $0 \%$ \\
\hline & & & nivo + BV & & $10 \%$ & $10 \%$ \\
\hline & & & ipili + nivo + BV & & $5 \%$ & $0 \%$ \\
\hline Davids et al. (2016) & RR hematol. malign. & $1 / 1 b$ & ipili & pneumonitis & $11 \%$ & $4 \%$ \\
\hline Bashey et al. (2009) & cancer post HSCT & 1 & ipili & pneumonitis & $7 \%$ & $7 \%$ \\
\hline \multirow[t]{2}{*}{ Maruyama et al. (2020) } & $\mathrm{RR} \mathrm{cHL}$ & 2 & nivo & IST lung disease & $24 \%$ & $6 \%$ \\
\hline & & & & pneumonitis & $12 \%$ & $0 \%$ \\
\hline Bröckelmann et al. (2020) & $\mathrm{ND} \mathrm{cHL}$ & 2 & nivo + AVD & pneumonitis & $1 \%$ & $0 \%$ \\
\hline Armand et al. (2018a) & $\mathrm{RR} \mathrm{cHL}$ & 2 & nivo & pneumonitis & $4 \%$ & $0 \%$ \\
\hline Herrera et al., 2018 & $\mathrm{RR} \mathrm{cHL}$ & $1 / 2$ & nivo + BV & pneumonitis & NA & $3 \%$ \\
\hline Lesokhin et al. (2016) & RR hematol. malign. & $1 b$ & nivo & pneumonitis & $11 \%$ & $4 \%$ \\
\hline Khodadoust et al. (2016) & RR MF, RR SS & 2 & pembro & pneumonitis & $8 \%$ & $4 \%$ \\
\hline Barta et al. (2019) & RR T-NHL & 2 & pembro & pneumonitis & $11 \%$ & $11 \%$ \\
\hline Armand et al. (2019) & RR PMBL & 2 & pembro & pneumonitis & $2 \%$ & $2 \%$ \\
\hline Armand et al. (2016) & $\mathrm{RR} \mathrm{cHL}$ & $1 b$ & pembro & pneumonitis & $10 \%$ & $0 \%$ \\
\hline \multirow[t]{2}{*}{ Liu et al. (2021) } & $\mathrm{RR} \mathrm{cHL}$ & 2 & camre & pneumonitis & $5 \%$ & $5 \%$ \\
\hline & & & camre + decitabine & & $5 \%$ & $0 \%$ \\
\hline Mei et al. (2020) & RR PMBL & 2 & camre + GVD & pneumonitis & $7 \%$ & $4 \%$ \\
\hline Usmani et al. (2019) & ND MM & 3 & pembro + lena + dex & pneumonitis & $1 \%$ & $0 \%$ \\
\hline Ribrag et al. (2019) & RR MM & $1 b$ & pembro & upper respir. tract inf. & $3 \%$ & $0 \%$ \\
\hline Mateos et al. (2019a) & RR MM & 3 & pembro + pom + dex & pneumonitis & $3 \%$ & $1 \%$ \\
\hline Badros et al. (2017) & $\mathrm{RR} \mathrm{MM}$ & 2 & pembro + pom + dex & pneumonitis & $13 \%$ & $2 \%$ \\
\hline Zeidan et al. (2018) & RR MDS & $1 b$ & ipili & pneumonitis & $3 \%$ & $0 \%$ \\
\hline Davids et al. (2020) & RR hematol. malign. & 1 & nivo & pneumonitis & $14 \%$ & $4 \%$ \\
\hline Daver et al. (2019) & $\mathrm{RR}$ AML & 2 & nivo + azacitidine & pneumonitis & $13 \%$ & $1 \%$ \\
\hline
\end{tabular}

RR, relapsed/refractory; ND, newly diagnosed; CHL, classical Hodgkin lymphoma; NHL, non-Hodgkin lymphoma; MM, multiple myeloma; PMBL, primary mediastinal large B cell lymphoma; DLBCL, diffuse large B cell lymphoma; MF, mycosis fungoides; SS, Sézary syndrome; AML, acute myeloid leukemia; MDS, myelodysplastic syndrome; HSCT, (allogeneic) hematopoietic stem cell transplantation; ipili, ipilimumab; liri, lirilumab; nivo, nivolumab; BV, brentuximab vedotin; AVD, adriamycin + vinblastine + dacarbazine; GVD, gemcitabine + vinorelbine + doxorubicin; pembro, pembrolizumab; pom, pomalidomide; dex, dexamethasone; ida, idarubucin; camre, camrelizumab; IST, interstitial.

tacrolimus. Patients experiencing grade 3-4 GI toxicity are not usually re-challenged with ICI therapy because of the high risk of relapse, but it could be discussed on an individual basis.

\section{PNEUMOTOXICITY}

Pulmonary toxicity of ICIs does not occur very often, but can be rapidly progressive and even fatal when present (Chhabra and Kennedy, 2021). Generally, it is more typical for anti-PD-(L)1 $\mathrm{mAbs}$ in comparison to anti-CTLA-4 inhibitors. Pneumonitis is one of the most common reasons for ICI discontinuation and in hematological malignancies, it is the leading treatment-related cause of death. Clinical symptoms include dyspnea, cough and chest pain and the median time to onset is 10-12 weeks after the initiation of ICI treatment.

The incidence of pneumotoxicity for ipilimumab monotherapy was $0-11 \%$, and $5-12 \%$ for the combination with nivolumab (Ansell et al., 2009; Davids et al., 2016; Diefenbach et al., 2020; Armand et al., 2021). There was one grade 5 case in each group (Davids et al., 2016; Diefenbach et al., 2020). Nivolumab and pembrolizumab caused pneumonitis/ interstitial lung disease/upper respiratory tract infection in $0-24 \%$ and $0-13 \%$ of patients, respectively (Ansell et al., 2015; Badros et al., 2017; Armand et al., 2019; Maruyama et al., 2020). There were 4 treatment-related deaths reported for the nivolumab cohort, while none for pembrolizumab (Lesokhin et al., 2016; Daver et al., 2019; Ramchandren et al., 2019; Diefenbach et al., 2020). No pneumotoxicity was observed in either of the anti-PD-L1 mAb clinical trials (avelumab, durvalumab + tremelimumab) (Kazandjian et al., 2021; Ribrag et al., 2021). (Table 5).

Pneumonitis is a diagnosis per exclusionem, so other conditions such as pulmonary embolism, pneumonia, viral infection or cancer progression should be excluded in the first place (Chhabra and Kennedy, 2021). The diagnostic method of choice is a high-resolution CT scan where ground glass opacities, organizing pneumonia, interstitial pneumonia or hypersensitivity pneumonitis could support the diagnosis of ICI-related lung toxicity (Haanen et al., 2017; Chhabra and Kennedy, 2021). Bronchoscopy with bronchoalveolar lavage may help to rule out infection. Lung biopsy is recommended only in dubious cases and it should be performed by video-assisted thoracoscopic surgery (Haanen et al., 2017; Delaunay et al., 2019). 
TABLE 6 | Incidence of rare irAEs in hematological malignancies.

\begin{tabular}{|c|c|c|c|c|c|c|}
\hline \multicolumn{7}{|c|}{ Rare Toxicities } \\
\hline \multirow[t]{2}{*}{ Author } & \multirow[t]{2}{*}{ Condition } & \multirow[t]{2}{*}{ Phase } & \multirow[t]{2}{*}{ Regimen } & \multirow[t]{2}{*}{ Symptom } & \multicolumn{2}{|c|}{ Incidence } \\
\hline & & & & & all grade & grade $\geq \mathbf{3}$ \\
\hline \multicolumn{7}{|l|}{ Cardiotoxicity } \\
\hline Mateos et al. (2019b) & $\mathrm{RR} \mathrm{MM}$ & 3 & pembro + pom + dex & myocarditis & $1 \%$ & $1 \%$ \\
\hline Usmani et al. (2019) & ND MM & 3 & pembro + lena + dex & myocarditis & $1 \%$ & $1 \%$ \\
\hline Younes et al. (2017) & ND FL & $1 \mathrm{~b} / 2$ & atezo + obi + benda & myocarditis & $3 \%$ & $3 \%$ \\
\hline \multicolumn{7}{|l|}{ Neurotoxicity } \\
\hline \multirow[t]{3}{*}{ Diefenbach et al. (2020) } & $\mathrm{RR} \mathrm{cHL}$ & $1 / 2$ & ipili + BV & peripheral sensory neuropathy & $69 \%$ & $4 \%$ \\
\hline & & & nivo + BV & & $53 \%$ & $0 \%$ \\
\hline & & & ipili + nivo + BV & & $36 \%$ & ०\% \\
\hline Cohen et al. (2021) & $\mathrm{RR} \mathrm{MM}$ & $2 / 3$ & cetreli + dara & autoimmune encephalitis & $11 \%$ & $0 \%$ \\
\hline D'Souza et al. (2019) & ND MM & 2 & pembro + lena & neuropathy & $7 \%$ & $0 \%$ \\
\hline Zeidan et al. (2018) & RR MDS & $1 b$ & ipili & Bell's palsy & $3 \%$ & $0 \%$ \\
\hline \multicolumn{7}{|l|}{ Other } \\
\hline Liu et al. (2021) & $\mathrm{RR} \mathrm{cHL}$ & 2 & camre + decitabine & myalgia & $10 \%$ & $0 \%$ \\
\hline \multirow[t]{2}{*}{ Shi et al. (2021) } & RR PTCL & 2 & geptanolimab & arthralgia & $2 \%$ & $0 \%$ \\
\hline & & & & Al hemolytic anemia & $1 \%$ & $1 \%$ \\
\hline Diefenbach et al. (2020) & $\mathrm{RR} \mathrm{cHL}$ & $1 / 2$ & ipili + nivo + BV & arthritis & $5 \%$ & $5 \%$ \\
\hline \multirow[t]{2}{*}{ Song et al. (2020) } & $\mathrm{RR} \mathrm{cHL}$ & 2 & tislelizumab & osteoarthritis & $1 \%$ & NA \\
\hline & & & & kidney injury & $1 \%$ & $1 \%$ \\
\hline Mei et al. (2020) & RR PMBL & 2 & camre + GVD & myalgia & $4 \%$ & $0 \%$ \\
\hline \multirow[t]{2}{*}{ Khodadoust et al. (2016) } & RR MF, RR SS & 2 & pembro & arthralgia + arthritis & $13 \%$ & $4 \%$ \\
\hline & & & & corneal ulcer & $4 \%$ & $4 \%$ \\
\hline Zinzani et al. (2019) & RR PMBL & $1 / 2$ & nivo + BV & kidney injury & $7 \%$ & $3 \%$ \\
\hline \multirow[t]{2}{*}{ Barta et al. (2019) } & RR T-NHL & 2 & pembro & vasculitis & $6 \%$ & $6 \%$ \\
\hline & & & & HLH & $6 \%$ & $6 \%$ \\
\hline Ansell et al. (2019) & RR DLBCL & 2 & nivo & nephritis + renal dysfunction & NA & $4 \%$ \\
\hline Armand et al. (2019) & RR PMBL & $1 b$ & pembro & myositis & $5 \%$ & $5 \%$ \\
\hline Davids et al. (2016) & RR hematol. malign. & $1 / 1 b$ & ipili & ITP & $4 \%$ & $0 \%$ \\
\hline Bashey et al. (2009) & cancer post HSCT & 1 & ipili & polyarthritis & $3 \%$ & $3 \%$ \\
\hline \multirow[t]{2}{*}{ D’Souza et al. (2019) } & ND MM & 2 & pembro + lena & arthralgia & $3 \%$ & $0 \%$ \\
\hline & & & & glomerulonephritis & $3 \%$ & $3 \%$ \\
\hline Davids et al. (2020) & RR hematol. malign. & 1 & nivo & arthralgia & $11 \%$ & $4 \%$ \\
\hline Daver et al. (2019) & RR AML & 2 & nivo + azacitidine & HLH & $1 \%$ & $1 \%$ \\
\hline Zeidan et al. (2018) & RR MDS & $1 b$ & ipili & myositis & $3 \%$ & $0 \%$ \\
\hline
\end{tabular}

RR, relapsed/refractory; ND, newly diagnosed; CHL, classical Hodgkin lymphoma; FL, follicular lymphoma; PTCL, peripheral T cell lymphoma; NHL- non-Hodgkin lymphoma; MM, multiple myeloma; PMBL, primary mediastinal large B cell lymphoma; DLBCL, diffuse large B cell lymphoma; MF, mycosis fungoides; SS, Sézary syndrome; AML, acute myeloid leukemia; MDS, myelodysplastic syndrome; HSCT, (allogeneic) hematopoietic stem cell transplantation; atezo, atezolizumab; ipili, ipilimumab; nivo, nivolumab; obi, obinutuzumab; benda, bendamustin; BV, brentuximab vedotin; GVD, gemcitabine + vinorelbine + doxorubicin; pembro, pembrolizumab; pom, pomalidomide; lena, lenalidomide; cetreli, cetrelimab; dara, daratumumab; dex, dexamethasone; ida, idarubucin; camre, camrelizumab; Al, autoimmune; HLH, hemophagocytic lymphohistiocytosis; ITP, immune thrombocytopenia.

Grade 1 pneumonitis is characterized by radiological findings without clinical symptoms (Haanen et al., 2017; Delaunay et al., 2019; Chhabra and Kennedy, 2021). These patients should be closely monitored and the delay of treatment considered. Oral prednisone $1 \mathrm{mg} / \mathrm{kg}$ and ICI interruption are recommended when the symptoms arise (grade 2). After recovery, steroids should be tapered over a period of 4-6 weeks due to possible relapse. Patients can be re-challenged with ICI when the dose of oral prednisone is $\leq 10 \mathrm{mg}$. Grade 3 and 4 cases of pneumonitis are associated with new or worsening hypoxia and lifethreatening breathing difficulties. These patients should be hospitalized and high-dose intravenous (methyl)prednisolone $(2-4 \mathrm{mg} / \mathrm{kg} /$ day) initiated. If there is no improvement after 2 days, infliximab $(5 \mathrm{mg} / \mathrm{kg})$, mycophenolate mofetil $(1000 \mathrm{mg}$ twice a day) or cyclophosphamide are to be added. Slow steroid tapering over at least 6 weeks is also recommended upon recovery to avoid relapse. In grade $\geq 3$ cases when the infectious etiology can't be quickly dismissed, broad-spectrum antibiotics are to be initiated alongside steroids. Restarting ICI therapy in patients experiencing severe pneumonitis (grade 3-4) is not recommended.

\section{RARE TOXICITIES}

\section{Cardiotoxicity}

Cardiac irAEs are associated with low incidence, but high mortality rates (Čelutkiené et al., 2020). They could present as 
pericarditis with pericardial pain, myocarditis with dyspnea due to pulmonary edema or arrhythmias with palpitations, presyncope and syncope (Tocchetti et al., 2019; Upadhrasta et al., 2019). Three cases of myocarditis were observed in hematooncological patients undergoing ICI therapy. Two were attributed to the combination of pembrolizumab with IMiD and dexamethasone and both of these events were fatal (Martinez-Calle et al., 2018; Mateos M.-V. et al., 2019; Usmani et al., 2019). The third was observed in a phase $1 \mathrm{~b} / 2$ clinical trial for newly diagnosed (ND) follicular lymphomas and investigated the combination of atezolizumab, obinutuzumab and bendamustine (Younes et al., 2017). In this case, the patient suffered from grade 4 myocarditis and bronchiolitis obliterans and the immediate cause of death was atezolizumab-related cardiac arrest preceded by an episode of dyspnea. (Table 6)

Diagnostic tools for myocarditis are cardiac biomarkers (troponin and creatinine kinase $\mathrm{MB}$ ), electrocardiogram, echocardiogram, cardiac MRI and endomyocardial biopsy (Upadhrasta et al., 2019; Pudil et al., 2020). Recently, global longitudinal strain (GLS) has also been considered to be of diagnostic and prognostic value as its decrease may be associated with ICI-related myocarditis (even in cases with preserved left ventricle ejection fraction) and higher probability of major adverse cardiac events (cardiogenic shock, arrest, complete heart block and cardiac death) (Awadalla et al., 2020; Čelutkiené et al., 2020). GLS is derived from transthoracic echocardiogram so no additional examination is required.

There are no definite treatment guidelines for ICI-associated cardiotoxicity. However, close monitoring of the patient and high-dose corticosteroids are essential in the management of these events. Steroid dosage varies from 1 to $2 \mathrm{mg} / \mathrm{kg}$ /day of prednisone in non-fulminant myocarditis to $1000 \mathrm{mg}$ of intravenous methylprednisolone per day in fulminant cases (Haanen et al., 2017; Upadhrasta et al., 2019). In nonresponders, escalation to infliximab, mycophenolate mofetil or anti-thymocyte globulin is recommended. The restart of ICI treatment may be considered only for patients experiencing grade 1 cardiotoxicity (Chhabra and Kennedy, 2021).

\section{Neurotoxicity}

Neurologic irAEs are uncommon, but have many forms. ICIassociated complications involving the peripheral nervous system include polyradiculopathies, neuropathies, myasthenic syndromes and myopathies, whereas the central nervous system can be affected by aseptic meningitis and encephalitis (Chhabra and Kennedy, 2021; Roth et al., 2021). Imaging, cerebrospinal fluid diagnostic, electroencephalography/ electromyoneurography and laboratory assessments help exclude tumor progression, vascular complications, infections, epilepsy, metabolic or toxic conditions and side effects of previous or current therapies. Median time to onset of these events ranges from 6 to 13 weeks (Haanen et al., 2017).

Relatively high incidence of mostly grade 1-2 peripheral sensory neuropathy was observed in a phase $1 / 2$ clinical trial investigating the combination of ipilimumab, nivolumab and BV for RR cHL patients (Diefenbach et al., 2020). However, these results should be regarded with caution due to the high neurotoxic potential of BV alone. D'Souza et al. reported a 7\% rate of neuropathy in their pembrolizumab-based study (D'Souza et al., 2019). Solitary and non-severe cases of Bell's palsy and autoimmune encephalitis occurred in blood cancer patients on ICI therapy (Zeidan et al., 2018; Cohen et al., 2021). (Table 6)

If neurological irAEs grade $\geq 2$ are considered, ICI treatment should be interrupted and systemic steroids initiated (Haanen et al., 2017; Chhabra and Kennedy, 2021; Roth et al., 2021). For patients experiencing grade 2 neurotoxicity, it is prednisolone $0.5-1 \mathrm{mg} / \mathrm{kg}$. For greater grades, intravenous (methyl) prednisolone $1-2 \mathrm{mg} / \mathrm{kg}$ is recommended. Plasmapheresis or intravenous immunoglobulins $(400 \mathrm{mg} / \mathrm{kg}$ for 5 days $)$ are indicated for non-responders to steroids with Guillain-Barré syndrome and myasthenia gravis. In the latter case, pyridoxine in an initial dose of $30 \mathrm{mg}$ three times a day is also advised. When the symptoms are not improving or worsening, other immunosuppressants such as azathioprin, cyclosporine, mycophenolate mofetil or even rituximab may be added, but there are no specific guidelines. Since these irAEs are potentially life-threatening, early consultation and cooperation with neurologists is appropriate.

\section{Rheumatological Toxicity}

Arthralgia and myalgia are the most frequently reported rheumatic irAEs, but arthritis, myositis and vasculitis were observed in blood cancer ICI trials as well (Bashey et al., 2009; Khodadoust et al., 2016; Zeidan et al., 2018; Armand et al., 2019; Barta et al., 2019; D’Souza et al., 2019; Davids et al., 2020; Diefenbach et al., 2020; Mei et al., 2020; Song et al., 2020; Liu et al., 2021; Shi et al., 2021). (Table 6) These adverse events are more likely to occur in ICIs acting on the PD-1 axis and may have later onset (Chhabra and Kennedy, 2021). Mild to moderate cases may be managed with analgesics or low-dose prednisolone (10-20 mg), more severe cases require cooperation with rheumatologists and higher doses of oral or intravenous corticosteroids or addition of other immunosuppressive agents such as infliximab, azathioprin, mycophenolate mofetil etc (Haanen et al., 2017; Jamal et al., 2020).

\section{Nephrotoxicity}

Rare irAEs also include renal impairments. The most common presentation is acute kidney injury (AKI) due to acute tubulointerstitial nephritis (Tinawi and Bastani, 2020; Chhabra and Kennedy, 2021). The mechanism is still unclear, but three risk factors were observed: 1) concomitant use of proton pump inhibitors, 2) low baseline estimated glomerular filtration rate $\left.\left(<30 \mathrm{ml} / \mathrm{min} / 1.73 \mathrm{~m}^{2}\right), 3\right)$ dual immune checkpoint blockade. Laboratory findings suggesting ICI-associated AKI could be microhematuria, subnephrotic range proteinuria, sterile pyuria and eosinophilia. Generally, median onset of AKI for anti-CTLA$4 \mathrm{mAbs}$ is 2 months and for PD-1 axis agents, 3-10 months. In our review of ICI blood cancer clinical trials, treatment-related $\mathrm{AKI} /$ nephritis occurred only in patients receiving anti-PD-1 treatment and one case was fatal (Ansell et al., 2019; Armand et al., 2019; Daver et al., 2019; D'Souza et al., 2019; Song et al., 2020). (Table 6) 
ICI therapy should be withheld in grade $\geq 2$ AKI (creatinine $>1.5 \mathrm{x}$ baseline/ULN) and may be restarted after restitution to grade 1, but more severe cases must be dealt with caution (Haanen et al., 2017; Tinawi and Bastani, 2020). Oral prednisolone $0.5-1 \mathrm{mg} / \mathrm{kg}$ should be initiated in grade $2 \mathrm{AKI}$ (creatinine $1.5-3 \mathrm{x}$ baseline/ULN) and intravenous (methyl) prednisolone $1-2 \mathrm{mg} / \mathrm{kg}$ in grade $\geq 3$ AKI (creatinine $>3 \mathrm{x}$ baseline/ULN). Kidney biopsy is not vital for determining the diagnosis, but may help to avoid unnecessary steroid treatment and ICI therapy discontinuation in dubious cases. Consultation with nephrologists would be appropriate.

\section{Other Toxicities}

Solitary cases of other types of irAEs were described in ICI blood cancer clinical trials. One patient with RR mycosis fungoides/ Sézary syndrome who was receiving pembrolizumab experienced grade 3 corneal ulcer (Khodadoust et al., 2016). Incidents of autoimmune hematologic toxicity included newly diagnosed immune thrombocytopenia during ipilimumab therapy, autoimmune hemolytic anemia grade 4 during geptanolimab therapy (anti-PD-1 mAb) and one grade 3 and one lethal case of hemophagocytic lymphohistiocytosis in clinical studies investigating pembrolizumab monotherapy in $\mathrm{T}$ cell lymphomas and the combination of nivolumab with azacitidine for acute myeloid leukemia patients, respectively (Davids et al., 2016; Barta et al., 2019; Daver et al., 2019; Shi et al., 2021). (Table 6)

\section{CONCLUSION}

Immune checkpoint blockade completely revolutionized the landscape of solid tumor oncology treatment. However, its journey in hemato-oncology has been much slower and more difficult. The initial great success in $\mathrm{CHL}$ and later PMBL, which led to accelerated FDA approval of nivolumab and pembrolizumab in these indications, was overshadowed by the general hold that was placed on large clinical trials investigating the promising combination of ICIs and IMiDs in the multiple myeloma setting due to suspicious treatment-related toxicity. Moreover, dual immune checkpoint blockade did not fulfil expectations and did not show increased benefit for patients suffering from hematological malignancies, only greater toxicity was reported. Aside from $\mathrm{cHL}$ and PMBL, ICI monotherapy did not prove to be effective either. Nevertheless, the future of ICIs in hemato-oncology seems to lie in combinations, either with conventional chemotherapy or other targeted agents such as mAbs, CAR T cells, PI3 $\kappa$ inhibitors, antibody-drug conjugates or bispecific antibodies as many ongoing studies and their promising efficacy results demonstrate. Therefore, hematooncologists should be prepared to work with ICIs more often and learn to recognize, diagnose and manage their specific irAEs.

Generally, the incidence and distribution of irAEs is approximately similar between solid tumor oncology and hemato-oncology. However, the exact rates may differ due to smaller amounts of treated patients and the fact that most of the safety data came from phase 1 and 2 clinical trials. The only available phase 3 safety data come from the controversial KEYNOTE-183 and KEYNOTE-185 studies (Mateos M.-V. et al., 2019; Usmani et al., 2019). The first one investigated the benefit of adding pembrolizumab to pomalidomide and dexamethasone in RR MM patients while the second one was for ND MM and studied lenalidomide and dexamethasone with or without pembrolizumab. These combinations proved toxic, when both clinical trials were suspended prematurely because of higher treatment-related mortality rates in experimental arms. Even if no specific adverse event of the ICI and IMiD combination has been unveiled to date, it is worth mentioning that the only two cases of grade 5 ICI-related myocarditis reported from prospective ICI blood cancer trials came from these two (1 from each). Investigation of another combination, daratumumab (anti-CD38 mAb) with PD-1 or PD-L1 inhibitors, was abandoned based on the unsatisfying results from a phase $1 \mathrm{~b} /$ 2 LUC2001 study of atezolizumab with or without daratumumab for advanced or metastatic non-small-cell lung cancer (NCT03023423) (Cohen et al., 2021). The cohort which received both agents did not reach superior outcome and there was also an imbalance of deaths in the experimental arm even if they weren't considered treatment-related. Although this study was targeting solid tumors, the decision of the investor affected all similar ongoing studies, including hemato-oncological (mainly MM again).

Agents acting on PD-1 axis are used in hemato-oncology more often than CTLA-4 inhibitors, so irAEs such as hyper/ hypothyroidism or pneumonitis may occur more frequently. Nevertheless, blood cancer specialists should be aware of all possible types of irAEs. Mostly, it is a diagnosis per exclusionem, so the physician is required to eliminate all other feasible causes. The majority of irAEs are mild to moderate, can be managed symptomatically or by oral steroids and mainly do not lead to permanent ICI discontinuation. On the other hand, fatal and potentially lethal irAEs were also reported, especially myocarditis, pneumonitis, neurologic events, hypophysitis, acute kidney injury and Stevens-Johnson syndrome. These events usually require permanent cessation of ICI treatment, rapid administration of high dose corticosteroids or other immunosuppressants (infliximab, mycophenolate mofetil, tacrloimus etc.) and multidisciplinary approach.

\section{AUTHOR CONTRIBUTIONS}

$\mathrm{KH}$ performed research and analyzed the data. $\mathrm{KH}$ and $\mathrm{TJ}$ wrote the manuscript. All authors read, critically reviewed and approved the manuscript.

\section{FUNDING}

This work has been supported the European Regional Development Fund-Project ENOCH (No. CZ.02.1.01/0.0/0.0/ 16_019/0000868), and the Ministry of Health of the Czech Republic (AZV 17-30089A), Institutional Support by MH CZDRO-FNOs/2019, MH CZ-DRO-FNOs/2020 Student's grant 
system SGS15/PrF/2021 University of Ostrava and by The Ministry of Education, Youth and Sports from the Large Infrastructures for Research, Experimental Development and Innovations project e-Infrastructure CZ-LM2018140.

\section{REFERENCES}

Ansell, S., Armand, P., Timmerman, J. M., Shipp, M. A., Bradley Garelik, M. B., Zhu, L., et al. (2015). Nivolumab in Patients (Pts) with Relapsed or Refractory Classical Hodgkin Lymphoma (R/R cHL): Clinical Outcomes from Extended Follow-Up of a Phase 1 Study (CA209-039). Blood 126, 583. doi:10.1182/ blood.v126.23.583.583

Ansell, S. M., Hurvitz, S. A., Koenig, P. A., LaPlant, B. R., Kabat, B. F., Fernando, D., et al. (2009). Phase I Study of Ipilimumab, an Anti-CTLA-4 Monoclonal Antibody, in Patients with Relapsed and Refractory B-Cell Non-hodgkin Lymphoma. Clin. Cancer Res. 15, 6446-6453. doi:10.1158/1078-0432.CCR-09-1339

Ansell, S. M., Minnema, M. C., Johnson, P., Timmerman, J. M., Armand, P., Shipp, M. A., et al. (2019). Nivolumab for Relapsed/Refractory Diffuse Large B-Cell Lymphoma in Patients Ineligible for or Having Failed Autologous Transplantation: A Single-Arm, Phase II Study. Jco 37, 481-489. doi:10.1200/JCO.18.00766

Armand, P., Engert, A., Younes, A., Lee, H. J., Santoro, A., Zinzani, P. L., et al. (2018a). Nivolumab for Relapsed or Refractory Classical Hodgkin Lymphoma (cHL) after Autologous Hematopoietic Cell Transplantation (Auto-HCT): Extended Follow-Up of the Phase 2 Single-Arm CheckMate 205 Study. Blood 132, 2897. doi:10.1182/blood-2018-99-112067

Armand, P., Lesokhin, A., Borrello, I., Timmerman, J., Gutierrez, M., Zhu, L., et al. (2021). A Phase 1b Study of Dual PD-1 and CTLA-4 or KIR Blockade in Patients with Relapsed/refractory Lymphoid Malignancies. Leukemia 35, 777-786. doi:10.1038/s41375-020-0939-1

Armand, P., Rodig, S. J., Melnichenko, V., Thieblemont, C., Bouabdallah, K., Tumyan, G., et al. (2018b). Pembrolizumab in Patients with Relapsed or Refractory Primary Mediastinal Large B-Cell Lymphoma (PMBCL): Data from the Keynote-013 and Keynote-170 Studies. Blood 132, 228. doi:10.1182/blood-2018-99-110220

Armand, P., Rodig, S., Melnichenko, V., Thieblemont, C., Bouabdallah, K., Tumyan, G., et al. (2019). Pembrolizumab in Relapsed or Refractory Primary Mediastinal Large B-Cell Lymphoma. Jco 37, 3291-3299. doi:10.1200/JCO.19.01389

Armand, P., Shipp, M. A., Ribrag, V., Michot, J.-M., Zinzani, P. L., Kuruvilla, J., et al. (2016). Pembrolizumab in Patients with Classical Hodgkin Lymphoma after Brentuximab Vedotin Failure: Long-Term Efficacy from the Phase $1 \mathrm{~b}$ Keynote-013 Study. Blood 128, 1108. doi:10.1182/blood.v128.22.1108.1108

Awadalla, M., Mahmood, S. S., Groarke, J. D., Hassan, M. Z. O., Nohria, A., Rokicki, A., et al. (2020). Global Longitudinal Strain and Cardiac Events in Patients with Immune Checkpoint Inhibitor-Related Myocarditis. J. Am. Coll. Cardiol. 75, 467-478. doi:10.1016/j.jacc.2019.11.049

Badros, A., Hyjek, E., Ma, N., Lesokhin, A., Dogan, A., Rapoport, A. P., et al. (2017). Pembrolizumab, Pomalidomide, and Low-Dose Dexamethasone for Relapsed/ refractory Multiple Myeloma. Blood 130, 1189-1197. doi:10.1182/blood-201703-775122

Barta, S. K., Zain, J., MacFarlane, A. W., Smith, S. M., Ruan, J., Fung, H. C., et al. (2019). Phase II Study of the PD-1 Inhibitor Pembrolizumab for the Treatment of Relapsed or Refractory Mature T-Cell Lymphoma. Clin. Lymphoma Myeloma Leuk. 19, 356-364.e3. doi:10.1016/j.clml.2019.03.022

Bashey, A., Medina, B., Corringham, S., Pasek, M., Carrier, E., Vrooman, L., et al. (2009). CTLA4 Blockade with Ipilimumab to Treat Relapse of Malignancy after Allogeneic Hematopoietic Cell Transplantation. Blood 113, 1581-1588. doi:10.1182/blood-2008-07-168468

Bröckelmann, P. J., Goergen, H., Keller, U., Meissner, J., Ordemann, R., Halbsguth, T. V., et al. (2020). Efficacy of Nivolumab and AVD in Early-Stage Unfavorable Classic Hodgkin Lymphoma. JAMA Oncol. 6, 872-880. doi:10.1001/ jamaoncol.2020.0750

Buchbinder, E. I., and Desai, A. (2016). CTLA-4 and PD-1 Pathways. Am. J. Clin. Oncol. 39, 98-106. doi:10.1097/COC.0000000000000239

\section{ACKNOWLEDGMENTS}

The authors would like to give thanks to Shira Timilsina Godfrey, M.D. for editing the article.

Cassaday, R. D., Garcia, K.-L. A., Fromm, J. R., Percival, M.-E. M., Turtle, C. J., Nghiem, P. T., et al. (2020). Phase 2 Study of Pembrolizumab for Measurable Residual Disease in Adults with Acute Lymphoblastic Leukemia. Blood Adv. 4, 3239-3245. doi:10.1182/bloodadvances.2020002403

Čelutkienè, J., Pudil, R., López-Fernández, T., Grapsa, J., Nihoyannopoulos, P., Bergler-Klein, J., et al. (2020). Role of Cardiovascular Imaging in Cancer Patients Receiving Cardiotoxic Therapies: a Position Statement on Behalf of the Heart Failure Association (HFA), the European Association of Cardiovascular Imaging (EACVI) and the Cardio-Oncology Council of the European Society of Cardiology (ESC). Eur. J. Heart Fail. 22, 1504-1524. doi:10.1002/ejhf.1957

Chang, L.-S., Barroso-Sousa, R., Tolaney, S. M., Hodi, F. S., Kaiser, U. B., and Min, L. (2019). Endocrine Toxicity of Cancer Immunotherapy Targeting Immune Checkpoints. Endocr. Rev. 40, 17-65. doi:10.1210/er.2018-00006

Chen, R., Zinzani, P. L., Fanale, M. A., Armand, P., Johnson, N. A., Brice, P., et al. (2017). Phase II Study of the Efficacy and Safety of Pembrolizumab for Relapsed/Refractory Classic Hodgkin Lymphoma. Jco 35, 2125-2132. doi:10.1200/JCO.2016.72.1316

Chhabra, N., and Kennedy, J. (2021). A Review of Cancer Immunotherapy Toxicity: Immune Checkpoint Inhibitors. J. Med. Toxicol. doi:10.1007/ s13181-021-00833-8

Cohen, Y. C., Oriol, A., Wu, K. L., Lavi, N., Vlummens, P., Jackson, C., et al. (2021). Daratumumab with Cetrelimab, an Anti-PD-1 Monoclonal Antibody, in Relapsed/Refractory Multiple Myeloma. Clin. Lymphoma Myeloma Leuk. 21, 46-54.e4. doi:10.1016/j.clml.2020.08.008

D'Souza, A., Hari, P., Pasquini, M., Braun, T., Johnson, B., Lundy, S., et al. (2019). A Phase 2 Study of Pembrolizumab during Lymphodepletion after Autologous Hematopoietic Cell Transplantation for Multiple Myeloma. Biol. Blood Marrow Transpl. 25, 1492-1497. doi:10.1016/j.bbmt.2019.04.005

Daver, N., Garcia-Manero, G., Basu, S., Boddu, P. C., Alfayez, M., Cortes, J. E., et al. (2019). Efficacy, Safety, and Biomarkers of Response to Azacitidine and Nivolumab in Relapsed/Refractory Acute Myeloid Leukemia: A Nonrandomized, Open-Label, Phase II Study. Cancer Discov. 9, 370-383. doi:10.1158/2159-8290.CD-18-0774

Davids, M. S., Kim, H. T., Bachireddy, P., Costello, C., Liguori, R., Savell, A., et al. (2016). Ipilimumab for Patients with Relapse after Allogeneic Transplantation. N. Engl. J. Med. 375, 143-153. doi:10.1056/NEJMoa1601202

Davids, M. S., Kim, H. T., Costello, C., Herrera, A. F., Locke, F. L., Maegawa, R. O., et al. (2020). A Multicenter Phase 1 Study of Nivolumab for Relapsed Hematologic Malignancies after Allogeneic Transplantation. Blood 135, 2182-2191. doi:10.1182/blood.2019004710

Delaunay, M., Prévot, G., Collot, S., Guilleminault, L., Didier, A., and Mazières, J. (2019). Management of Pulmonary Toxicity Associated with Immune Checkpoint Inhibitors. Eur. Respir. Rev. 28, 190012. doi:10.1183/16000617.0012-2019

Diefenbach, C. S., Hong, F., Ambinder, R. F., Cohen, J. B., Robertson, M. J., David, K. A., et al. (2020). Ipilimumab, Nivolumab, and Brentuximab Vedotin Combination Therapies in Patients with Relapsed or Refractory Hodgkin Lymphoma: Phase 1 Results of an Open-Label, Multicentre, Phase 1/2 Trial. Lancet Haematol. 7, e660-e670. doi:10.1016/S2352-3026(20)30221-0

Fan, Y., Xie, W., Huang, H., Wang, Y., Li, G., Geng, Y., et al. (2021). Association of Immune Related Adverse Events with Efficacy of Immune Checkpoint Inhibitors and Overall Survival in Cancers: A Systemic Review and MetaAnalysis. Front. Oncol. 11, 633032. doi:10.3389/fonc.2021.633032

Haanen, J. B. a. G., Carbonnel, F., Robert, C., Kerr, K. M., Peters, S., Larkin, J., et al. (2017). Management of Toxicities from Immunotherapy: ESMO Clinical Practice Guidelines for Diagnosis, Treatment and Follow-Up. Ann. Oncol. 28, iv119-iv142. doi:10.1093/annonc/mdx225

Herrera, A. F., Moskowitz, A. J., Bartlett, N. L., Vose, J. M., Ramchandren, R., Feldman, T. A., et al. (2018). Interim Results of Brentuximab Vedotin in Combination with Nivolumab in Patients with Relapsed or Refractory Hodgkin Lymphoma. Blood 131, 1183-1194. doi:10.1182/blood-2017-10-811224 
Hradska, K., Kascak, M., Hajek, R., and Jelinek, T. (2020). Identifying and Treating Candidates for Checkpoint Inhibitor Therapies in Multiple Myeloma and Lymphoma. Expert Rev. Hematol. 13, 375-392. doi:10.1080/17474086.2020.1733405

Jamal, S., Hudson, M., Fifi-Mah, A., and Ye, C. (2020). Immune-related Adverse Events Associated with Cancer Immunotherapy: A Review for the Practicing Rheumatologist. J. Rheumatol. 47, 166-175. doi:10.3899/ jrheum.190084

Jelinek, T., and Hajek, R. (2016). PD-1/PD-L1 Inhibitors in Multiple Myeloma: The Present and the Future. Oncoimmunology 5, e1254856. doi:10.1080/ 2162402X.2016.1254856

Jelinek, T., Mihalyova, J., Kascak, M., Duras, J., and Hajek, R. (2017). PD-1/PD-L1 Inhibitors in Haematological Malignancies: Update 2017. Immunology 152, 357-371. doi:10.1111/imm.12788

Jelinek, T., Paiva, B., and Hajek, R. (2018). Update on PD-1/pd-L1 Inhibitors in Multiple Myeloma. Front. Immunol. 9, 2431. doi:10.3389/ fimmu.2018.02431

Kazandjian, D., Dew, A., Hill, E., Ramirez, E. G., Morrison, C., Mena, E., et al. (2021). Avelumab, a PD-L1 Inhibitor, in Combination with Hypofractionated Radiotherapy and the Abscopal Effect in Relapsed Refractory Multiple Myeloma. Oncol. 26, 288-e541. doi:10.1002/onco.13712

Khodadoust, M., Rook, A. H., Porcu, P., Foss, F. M., Moskowitz, A. J., Shustov, A. R., et al. (2016). Pembrolizumab for Treatment of Relapsed/ Refractory Mycosis Fungoides and Sezary Syndrome: Clinical Efficacy in a Citn Multicenter Phase 2 Study. Blood 128, 181. doi:10.1182/ blood.v128.22.181.181

Khouri, I. F., Fernandez Curbelo, I., Turturro, F., Jabbour, E. J., Milton, D. R., Bassett, R. L., et al. (2018). Ipilimumab Plus Lenalidomide after Allogeneic and Autologous Stem Cell Transplantation for Patients with Lymphoid Malignancies. Clin. Cancer Res. 24, 1011-1018. doi:10.1158/10780432.CCR-17-2777

Lesokhin, A. M., Ansell, S. M., Armand, P., Scott, E. C., Halwani, A., Gutierrez, M., et al. (2016). Nivolumab in Patients with Relapsed or Refractory Hematologic Malignancy: Preliminary Results of a Phase Ib Study. Jco 34, 2698-2704. doi:10.1200/JCO.2015.65.9789

Liu, Y., Wang, C., Li, X., Dong, L., Yang, Q., Chen, M., et al. (2021). Improved Clinical Outcome in a Randomized Phase II Study of Anti-PD-1 Camrelizumab Plus Decitabine in Relapsed/refractory Hodgkin Lymphoma. J. Immunother. Cancer 9, e002347. doi:10.1136/jitc-2021-002347

Martinez-Calle, N., Rodriguez-Otero, P., Villar, S., Mejías, L., Melero, I., Prosper, F., et al. (2018). Anti-PD1 Associated Fulminant Myocarditis after a Single Pembrolizumab Dose: the Role of Occult Pre-existing Autoimmunity. Haematologica 103, e318-e321. doi:10.3324/ haematol.2017.185777

Maruyama, D., Terui, Y., Yamamoto, K., Fukuhara, N., Choi, I., Kuroda, J., et al. (2020). Final Results of a Phase II Study of Nivolumab in Japanese Patients with Relapsed or Refractory Classical Hodgkin Lymphoma. Jpn. J. Clin. Oncol. 50, 1265-1273. doi:10.1093/jjco/hyaa117

Mateos, M.-V., Blacklock, H., Schjesvold, F., Oriol, A., Simpson, D., George, A., et al. (2019a). Pembrolizumab Plus Pomalidomide and Dexamethasone for Patients with Relapsed or Refractory Multiple Myeloma (KEYNOTE-183): a Randomised, Open-Label, Phase 3 Trial. The Lancet Haematol. 6 (9), e459-e469. doi:10.1016/S2352-3026(19)30110-3

Mateos, M. V., Orlowski, R. Z., Ocio, E. M., Rodríguez-Otero, P., Reece, D., Moreau, P., et al. (2019b). Pembrolizumab Combined with Lenalidomide and Low-dose Dexamethasone for Relapsed or Refractory Multiple Myeloma: Phase I KEYNOTE -023 Study. Br. J. Haematol. 186. doi:10.1111/bjh.15946

Matsuki, E., and Younes, A. (2016). Checkpoint Inhibitors and Other Immune Therapies for Hodgkin and Non-hodgkin Lymphoma. Curr. Treat. Options. Oncol. 17, 31. doi:10.1007/s11864-016-0401-9

Mei, Q., Zhang, W., Liu, Y., Yang, Q., Rasko, J. E. J., Nie, J., et al. (2020). Camrelizumab Plus Gemcitabine, Vinorelbine, and Pegylated Liposomal Doxorubicin in Relapsed/Refractory Primary Mediastinal B-Cell Lymphoma: A Single-Arm, Open-Label, Phase II Trial. Clin. Cancer Res. 26, 4521-4530. doi:10.1158/1078-0432.CCR-200514

Pudil, R., Mueller, C., Čelutkiené, J., Henriksen, P. A., Lenihan, D., Dent, S., et al. (2020). Role of Serum Biomarkers in Cancer Patients Receiving Cardiotoxic
Cancer Therapies: a Position Statement from the Cardio-Oncology Study Group of the Heart Failure Association and the Cardio-Oncology Council of the European Society of Cardiology. Eur. J. Heart Fail. 22, 1966-1983. doi:10.1002/ejhf.2017

Ramchandren, R., Domingo-Domènech, E., Rueda, A., Trněný, M., Feldman, T. A., Lee, H. J., et al. (2019). Nivolumab for Newly Diagnosed Advanced-Stage Classic Hodgkin Lymphoma: Safety and Efficacy in the Phase II CheckMate 205 Study. Jco 37, 1997-2007. doi:10.1200/JCO.19.00315

Ransohoff, J. D., and Kwong, B. Y. (2017). Cutaneous Adverse Events of Targeted Therapies for Hematolymphoid Malignancies. Clin. Lymphoma Myeloma Leuk. 17, 834-851. doi:10.1016/j.clml.2017.07.005

Ravandi, F., Assi, R., Daver, N., Benton, C. B., Kadia, T., Thompson, P. A., et al. (2019). Idarubicin, Cytarabine, and Nivolumab in Patients with Newly Diagnosed Acute Myeloid Leukaemia or High-Risk Myelodysplastic Syndrome: a Single-Arm, Phase 2 Study. Lancet Haematol. 6, e480-e488. doi:10.1016/S2352-3026(19)30114-0

Ribrag, V., Avigan, D. E., Green, D. J., Wise-Draper, T., Posada, J. G., Vij, R., et al. (2019). Phase $1 \mathrm{~b}$ Trial of Pembrolizumab Monotherapy for Relapsed/refractory Multiple Myeloma: KEYNOTE -013. Br. J. Haematol. 186, e41-e44. doi:10.1111/bjh.15888

Ribrag, V., Lee, S. T., Rizzieri, D., Dyer, M. J. S., Fayad, L., Kurzrock, R., et al. (2021). A Phase 1b Study to Evaluate the Safety and Efficacy of Durvalumab in Combination with Tremelimumab or Danvatirsen in Patients with Relapsed or Refractory Diffuse Large B-Cell Lymphoma. Clin. Lymphoma Myeloma Leuk. 21, 309-317. e3. doi:10.1016/ j.clml.2020.12.012

Rocha, M., Correia de Sousa, J., Salgado, M., Araújo, A., and Pedroto, I. (2019). Management of Gastrointestinal Toxicity from Immune Checkpoint Inhibitor. GE Port J. Gastroenterol. 26, 268-274. doi: $10.1159 / 000494569$

Roth, P., Winklhofer, S., Müller, A. M. S., Dummer, R., Mair, M. J., Gramatzki, D., et al. (2021). Neurological Complications of Cancer Immunotherapy. Cancer Treat. Rev. 97, 102189. doi:10.1016/ j.ctrv.2021.102189

Shi, Y., Wu, J., Wang, Z., Zhang, L., Wang, Z., Zhang, M., et al. (2021). Efficacy and Safety of Geptanolimab (GB226) for Relapsed or Refractory Peripheral T Cell Lymphoma: an Open-Label Phase 2 Study (Gxplore-002). J. Hematol. Oncol. 14, 12. doi:10.1186/s13045-021-01033-1

Smyth, M. J., and Teng, M. W. (2018). 2018 Nobel Prize in Physiology or Medicine. Clin. Transl Immunol. 7, e1041. doi:10.1002/cti2.1041

Song, Y., Gao, Q., Zhang, H., Fan, L., Zhou, J., Zou, D., et al. (2020). Treatment of Relapsed or Refractory Classical Hodgkin Lymphoma with the Anti-PD-1, Tislelizumab: Results of a Phase 2, Single-Arm, Multicenter Study. Leukemia 34, 533-542. doi:10.1038/s41375-019-0545-2

Tian, Y., Abu-Sbeih, H., and Wang, Y. (2018). Immune Checkpoint InhibitorsInduced Hepatitis. Adv. Exp. Med. Biol. 995, 159-164. doi:10.1007/978-3030-02505-2_8

Tinawi, M., and Bastani, B. (2020). Nephrotoxicity of Immune Checkpoint Inhibitors: Acute Kidney Injury and beyond. Cureus 12, e12204. doi:10.7759/ cureus. 12204

Tocchetti, C. G., Cadeddu, C., Di Lisi, D., Femminò, S., Madonna, R., Mele, D., et al. (2019). From Molecular Mechanisms to Clinical Management of Antineoplastic Drug-Induced Cardiovascular Toxicity: A Translational Overview. Antioxid. Redox Signaling 30, 2110-2153. doi:10.1089/ ars.2016.6930

Tuscano, J. M., Maverakis, E., Groshen, S., Tsao-Wei, D., Luxardi, G., Merleev, A. A., et al. (2019). A Phase I Study of the Combination of Rituximab and Ipilimumab in Patients with Relapsed/Refractory B-Cell Lymphoma. Clin Cancer Res. 25, 7004-7013. doi:10.1158/1078-0432.CCR-19-0438

Upadhrasta, S., Elias, H., Patel, K., and Zheng, L. (2019). Managing Cardiotoxicity Associated with Immune Checkpoint Inhibitors. Chronic Dis. Translational Med. 5, 6-14. doi:10.1016/j.cdtm.2019.02.004

Usmani, S. Z., Schjesvold, F., Oriol, A., Karlin, L., Cavo, M., Rifkin, R. M., et al. (2019). Pembrolizumab Plus Lenalidomide and Dexamethasone for Patients with Treatment-Naive Multiple Myeloma (KEYNOTE185): a Randomised, Open-Label, Phase 3 Trial. Lancet Haematol. 6 (9), e448-e458. doi:10.1016/S2352-3026(19)30109-7 
Wang, Y., Wu, L., Tian, C., and Zhang, Y. (2018). PD-1-PD-L1 ImmuneCheckpoint Blockade in Malignant Lymphomas. Ann. Hematol. 97, 229-237. doi:10.1007/s00277-017-3176-6

Younes, A., Brody, J., Carpio, C., Lopez-Guillermo, A., Ben-Yehuda, D., Ferhanoglu, B., et al. (2019). Safety and Activity of Ibrutinib in Combination with Nivolumab in Patients with Relapsed Nonhodgkin Lymphoma or Chronic Lymphocytic Leukaemia: a Phase 1/2a Study. Lancet Haematol. 6, e67-e78. doi:10.1016/S2352-3026(18)30217-5

Younes, A., John, B. M., Diefenbach, C. S., Ferrari, S., Kahn, C., Sharman, J. P., et al. (2017). Safety and Efficacy of Atezolizumab in Combination with Obinutuzumab and Bendamustine in Patients with Previously Untreated Follicular Lymphoma: An Interim Analysis. Blood 130, 481.

Zeidan, A. M., Knaus, H. A., Robinson, T. M., Towlerton, A. M. H., Warren, E. H., Zeidner, J. F., et al. (2018). A Multi-center Phase I Trial of Ipilimumab in Patients with Myelodysplastic Syndromes Following Hypomethylating Agent Failure. Clin. Cancer Res. 24, 3519-3527. doi:10.1158/1078-0432.CCR-17-3763

Zinzani, P. L., Santoro, A., Gritti, G., Cunningham, D., Johnsone, N. A., Manleyh, T. Francis. S., et al. (2019). Nivolumab Combined with Brentuximab Vedotin for Relapsed/Refractory Primary Mediastinal Large B-Cell Lymphoma: Efficacy and Safety from the Phase II CheckMate 436 Study. Jco 37, 3081-3089. doi:10.1200/JCO.19.01492

Conflict of Interest: The authors declare that the research was conducted in the absence of any commercial or financial relationships that could be construed as a potential conflict of interest.

Publisher's Note: All claims expressed in this article are solely those of the authors and do not necessarily represent those of their affiliated organizations, or those of the publisher, the editors and the reviewers. Any product that may be evaluated in this article, or claim that may be made by its manufacturer, is not guaranteed or endorsed by the publisher.

Copyright (C) 2021 Hradska, Hajek and Jelinek. This is an open-access article distributed under the terms of the Creative Commons Attribution License (CC BY). The use, distribution or reproduction in other forums is permitted, provided the original author(s) and the copyright owner(s) are credited and that the original publication in this journal is cited, in accordance with accepted academic practice. No use, distribution or reproduction is permitted which does not comply with these terms. 